\title{
Owners of Nuclear Power Plants
}

\author{
RECEIVED
}

NOV 251996

OSTI

\section{Prepared by}

C. R. Hudson, V. S. White

Oak Ridge National Laboratory

Prepared for

U.S. Nuclear Regulatory Commission 


\section{AVAILABILITY NOTICE}

Availability of Reference Materials Cited in NRC Publications

Most documents cited in NRC publications will be available from one of the following sources:

1. The NRC Public Document Room, 2120 L Street, NW., Lower Level, Washington, DC 20555-0001

2. The Superintendent of Documents, U.S. Government Printing Office, P. O. Box 37082, Washington, DC 20402-9328

3. The National Technical Information Service, Springfield, VA 22161-0002

Although the listing that follows represents the majority of documents cited in NRC publications, it is not intended to be exhaustive.

Referenced documents available for inspection and copying for a fee from the NRC Public Document Room include NRC correspondence and internal NRC memoranda; NRC bulletins, circulars. information notices, inspection and investigation notices; licensee event reports; vendor reports and correspondence: Commission papers; and applicant and licensee documents and correspondence.

The following documents in the NUREG series are available for purchase from the Government Printing Office: formal NRC staff and contractor reports, NRC-sponsored conference proceedings, international agreement reports, grantee reports, and NRC booklets and brochures. Also available are regulatory guides, NRC regulations in the Code of Federal Regulations, and Nuclear Regulatory Commission Issuances.

Documents avallable from the National Technical Information Service include NUREG-series reports and technical reports prepared by other Federal agencies and reports prepared by the Atomic Energy Commission, forerunner agency to the Nuclear Regulatory Commission.

Documents available from public and special technical libraries include all open literature items, such as books. journal articles. and transactions. Federal Register notices. Federal and State legislation, and congressional reports can usually be obtained from these libraries.

Documents such as theses, dissertations, foreign reports and translations, and non-NRC conference proceedings are available for purchase from the organization sponsoring the publication cited.

Single copies of NRC draft reports are available free. to the extent of supply. upon written request to the Office of Administration, Distribution and Mail Services Section, U.S. Nuclear Regulatory Commission, Washington, DC 20555-0001.

Coples of industry codes and standards used in a substantive manner in the NRC regulatory process are maintained at the NRC Library. Two White Flint North, 11545 Rockville Pike, Rockville, MD 20852-2738, for use by the public. Codes and standards are usually copyrighted and may be purchased from the originating organization or, if they are American National Standards, from the American National Standards Institute. 1430 Broadway, New York, NY 10018-3308.

\section{DISCLAIMER NOTICE}

This report was prepared as an account of work sponsored by an agency of the United States Government. Neither the United States Government nor any agency thereof, nor any of their employees, makes any warranty, expressed or implied, or assumes any legal liability or responsibility for any third party's use, or the results of such use, of any information, apparatus, product, or process disclosed in this report, or represents that its use by such third party would not infringe privately owned rights. 


\section{Owners of Nuclear Power Plants}

Manuscript Completed: October 1996

Date Published: November 1996

Prepared by

C. R. Hudson, V. S. White

Oak Ridge National Laboratory

Managed by Lockheed Martin Energy Research Corporation

P.O. Box 2009

Oak Ridge, TN 37831-8038

R. Wood, NRC Project Manager

Prepared for

Division of Reactor Program Management

Office of Nuclear Reactor Regulation

U.S. Nuclear Regulatory Commission

Washington, DC 20555-0001

NRC Job Code J2411 


\section{DISCLAIMER}

Portions of this document may be illegible in electronic image products. Images are produced from the best available original document. 


\section{DISCLAIMER}

This report was prepared as an account of work sponsored by an agency of the United States Government. Neither the United States Government nor any agency thereof, nor any of their employees, makes any warranty, express or implied, or assumes any legal liability or responsibility for the accuracy, completeness, or usefulness of any information, apparatus, product, or process disclosed, or represents that its use would not infringe privately owned rights. Reference herein to any specific commercial product, process, or service by trade name, trademark, manufacturer, or otherwise does not necessarily constitute or imply its endorsement, recommendation, or favoring by the United States Government or any agency thereof. The views and opinions of authors expressed herein do not necessarily state or reflect those of the United States Government or any agency thereof. 


\section{ABSTRACT}

Commercial nuclear power plants in this country can be owned by a number of separate entities, each with varying ownership proportions. Each of these owners may, in turn, have a parent/subsidiary relationship to other companies. In addition, the operator of the plant may be a different entity as well. This report provides a compilation on the owners/operators for all commercial power reactors in the United States. While the utility industry is currently experiencing changes in organizational structure which may affect nuclear plant ownership, the data in this report is current as of July 1996. The report is divided into sections representing different aspects of nuclear plant ownership. 


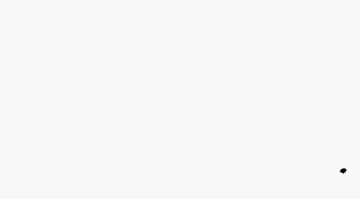




\section{CONTENTS}

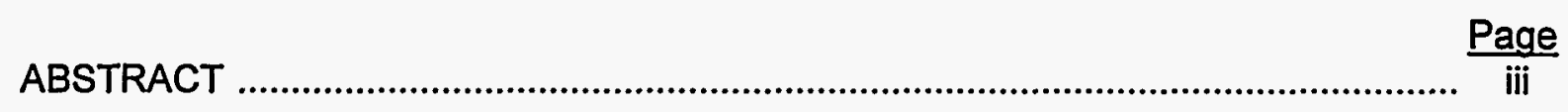

LIST OF TABLES ............................................................................................ vii

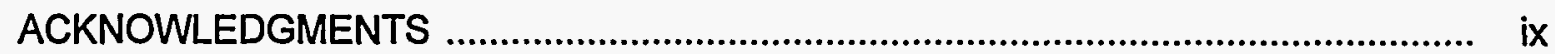

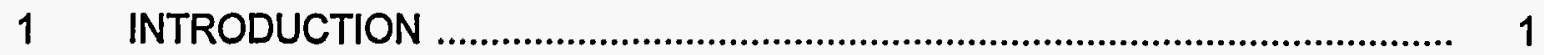

2 NUCLEAR POWER PLANT PERCENTAGE OWNERSHIP

ORDERED BY PLANT NAME ................................................................... 3

3 NUCLEAR POWER PLANT PERCENTAGE OWNERSHIP

ORDERED BY UTILITY NAME ............................................................ 15

4 UTILITYICOMPANY RELATIONSHIPS ORDERED BY

PARENT/HOLDING COMPANY .......................................................... 27

5 UTILITYICOMPANY RELATIONSHIPS ORDERED BY

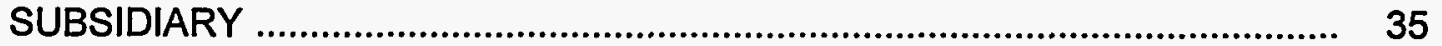

$6 \quad$ NUCLEAR POWER PLANTS LISTED BY OPERATOR .................................. 43

$7 \quad$ NUCLEAR POWER PLANT OPERATORS LISTED BY PLANT NAME .......... 49

$8 \quad$ NUCLEAR POWER PLANTS LISTED BY STATE ......................................... 55

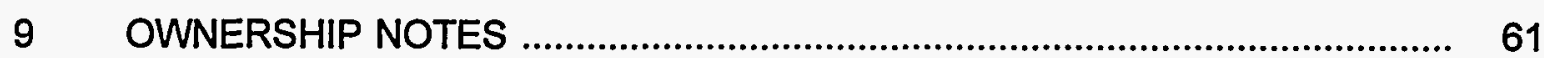

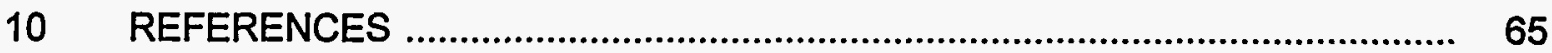




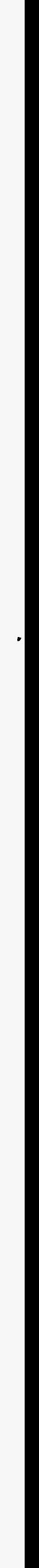




\section{LIST OF TABLES}

Table

Page

1. Nuclear power plant percentage ownership by plant ................................................ 5

2. Nuclear power plant percentage ownership by utility .............................................. 17

3. Utility/company relationships by parent/holding company ........................................ 29

4. Utility/company relationships by subsidiary .............................................................. 37

5. Nuclear power plants listed by operator .................................................................. 45

6. Nuclear power plant operators listed by plant ......................................................... 51

7. Nuclear power plants by state ................................................................................... 57

8. Ownership notes ..................................................................................................... 63 


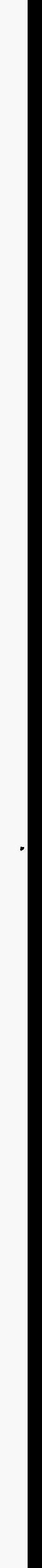




\section{ACKNOWLEDGMENTS}

The authors wish to thank the numerous utility company personnel who were contacted in order to assist in determining and/or confirming the ownership data contained in this report. In addition, the authors express their appreciation to Robert Wood, Technical Monitor, Division of Reactor Program Management, Office of Nuclear Reactor Regulation, U.S. Nuclear Regulatory Commission for his insight and guidance. 


\section{INTRODUCTION}

Commercial nuclear power plants in this country can be owned by a number of separate entities, each with varying ownership proportions. Each of these owners may, in turn, have a parent/subsidiary relationship to other companies. In addition, the operator of the plant may be a different entity as well. This report provides a compilation on the owners/operators for all commercial power reactors in the United States. While the utility industry is currently experiencing changes in organizational structure which may affect nuclear plant ownership, the data in this report is current as of July 1996. The report is divided into sections representing different aspects of nuclear plant ownership. 


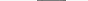


2 NUCLEAR POWER PLANT PERCENTAGE OWNERSHIP ORDERED BY PLANT NAME 

Table 1. Nuclear power plant percentage ownership by plant

\begin{tabular}{|c|c|c|c|c|}
\hline Plant name & Location & $\begin{array}{l}\text { Operating } \\
\text { status }\end{array}$ & Ownership & $\begin{array}{l}\text { Ownership } \\
\text { percentage }\end{array}$ \\
\hline Arkansas Nuclear One, Units $1 \& 2$ & Arkansas & FPL & Entergy Arkansas, Inc. & 100.0 \\
\hline Arnold (Duane) Energy Center & lowa & FPL & $\begin{array}{l}\text { IES Utilities, Inc. } \\
\text { Central lowa Power Cooperative } \\
\text { Com Belt Power Cooperative }\end{array}$ & $\begin{array}{l}70.0 \\
20.0 \\
10.0\end{array}$ \\
\hline Beaver Valley Power Station, Unit 1 & Pennsylvania & FPL & $\begin{array}{l}\text { Duquesne Light Company } \\
\text { Ohio Edison Company } \\
\text { Pennsylvania Power Company }\end{array}$ & $\begin{array}{l}47.5 \\
35.0 \\
17.5\end{array}$ \\
\hline Beaver Valley Power Station, Unit 2 & Pennsylvania & FPL & $\begin{array}{l}\text { Ohio Edison Company } \\
\text { Cleveland Electric Illuminating Company } \\
\text { Toledo Edison Company } \\
\text { Duquesne Light Company }\end{array}$ & $\begin{array}{l}41.88 \\
24.47 \\
19.91 \\
13.74\end{array}$ \\
\hline Bellefonte Nuclear Plant, Units $1 \& 2$ & Alabama & $\mathrm{CDH}$ & Tennessee Valley Authority & 100.0 \\
\hline Big Rock Point Nuclear Plant & Michigan & FPL & Consumers Power Company & 100.0 \\
\hline Braidwood Station, Units 1 \& 2 & Illinois & FPL & Commonwealth Edison Company & 100.0 \\
\hline Brown's Ferry Nuclear Power Station, Unit 1 & Alabama & SDUR & Tennessee Valley Authority & 100.0 \\
\hline Brown's Ferry Nuclear Power Station, Units 2 \& 3 & Alabama & FPL & Tennessee Valley Authority & 100.0 \\
\hline Brunswick Steam Electric Plant, Units 1 \& 2 & North Carolina & FPL & $\begin{array}{l}\text { Carolina Power \& Light Company } \\
\text { North Carolina Eastern Municipal Power Agency }\end{array}$ & $\begin{array}{l}81.67 \\
18.33\end{array}$ \\
\hline Byron Station, Units 1 \& 2 & Illinois & FPL & Commonwealth Edison Company & 100.0 \\
\hline Callaway Plant & Missouri & FPL & Union Electric Company & 100.0 \\
\hline Calvert Cliffs Nuclear Power Plant, Units 1 \& 2 & Maryland & FPL & Baltimore Gas \& Electric Company & 100.0 \\
\hline
\end{tabular}




\begin{tabular}{|c|c|c|c|c|}
\hline Plant name & Location & $\begin{array}{l}\text { Operating } \\
\text { status }^{*}\end{array}$ & Ownership & $\begin{array}{l}\text { Ownership } \\
\text { percentage }\end{array}$ \\
\hline \multirow[t]{3}{*}{ Catawba Nuclear Station, Unit 1} & South Carolina & FPL & North Carolina Electric Membership Corporation & 56.25 \\
\hline & & & Duke Power Company & 25.00 \\
\hline & & & Saluda River Electric Cooperative & 18.75 \\
\hline \multirow[t]{2}{*}{ Catawba Nuclear Station, Unit 2} & South Carolina & FPL & North Carolina Eastern Municipal Power Agency & 75.0 \\
\hline & & & Piedmont Municipal Power Agency & 25.0 \\
\hline \multirow[t]{2}{*}{ Clinton Power Station } & Illinois & FPL & Illinois Power Company & 86.79 \\
\hline & & & Soyland Power Cooperative, Inc. & 13.21 \\
\hline Comanche Peak Steam Electric Station, Units 1 \& 2 & Texas & FPL & TU Electric Company & 100.0 \\
\hline Cook (Donald C.) Nuclear Power Plant, Units 1 \& 2 & Michigan & FPL & Indiana Michigan Power Company & 100.0 \\
\hline Cooper Nuclear Station & Nebraska & FPL & Nebraska Public Power District & 100.0 \\
\hline \multirow[t]{11}{*}{ Crystal River Nuclear Plant, Unit 3} & Florida & FPL & Florida Power Corporation & 90.4473 \\
\hline & & & Seminole Electric Cooperative, Inc. & 1.6994 \\
\hline & & & Orlando Utilities Commission & 1.6015 \\
\hline & & & Gainesville (City of) Regional Utilities & 1.4079 \\
\hline & & & Ocala (City of) Utilities Division & 1.3333 \\
\hline & & & Tallahassee (City of) Electric Department & 1.3333 \\
\hline & & & Leesburg (City of) Municipal Electric Department & 0.8244 \\
\hline & & & Kissimee (City of) Utilities & 0.6754 \\
\hline & & & New Smyrna Beach Utilities Commission & 0.5608 \\
\hline & & & Alachua (City of) Electric Department & 0.0779 \\
\hline & & & Bushnell (City of) Utility Department & 0.0388 \\
\hline \multirow[t]{2}{*}{ Davis-Besse Nuclear Power Station } & Ohio & FPL & Cleveland Electric Illuminating Company & 51.38 \\
\hline & & & Toledo Edison Company & 48.62 \\
\hline Diablo Canyon Nuclear Power Plant, Units 1 \& 2 & California & FPL & Pacific Gas \& Electric Company & 100.0 \\
\hline
\end{tabular}


Table 1 (continued)

\begin{tabular}{|c|c|c|c|c|}
\hline Plant name & Location & $\begin{array}{l}\text { Operating } \\
\text { status }{ }^{*}\end{array}$ & Ownership & $\begin{array}{l}\text { Ownership } \\
\text { percentage }\end{array}$ \\
\hline Dresden Nuclear Power Station, Unit 1 & Illinois & POL & Commonwealth Edison Company & 100.0 \\
\hline Dresden Nuclear Power Station, Units 2 \& 3 & Illinois & FPL & Commonwealth Edison Company & 100.0 \\
\hline Farley (Joseph M.) Nuclear Plant, Units 1 \& 2 & Alabama & FPL & Alabama Power Company & 100.0 \\
\hline Fermi (Enrico) Atomic Power Plant, Unit 1 & Michigan & POL & Power Reactor Development Company & 100.0 \\
\hline Fermi (Enrico) Atomic Power Plant, Unit 2 & Michigan & FPL & Detroit Edison Company & 100.0 \\
\hline Fitzpatrick (James A.) Nuclear Power Plant & New York & FPL & New York Power Authority & 100.0 \\
\hline Fort Calhoun Station & Nebraska & FPL & Omaha Public Power District & 100.0 \\
\hline Fort St. Vrain Nuclear Generating Station & Colorado & POL & Public Service Company of Colorado & 100.0 \\
\hline Ginna (Robert E.) Nuclear Power Plant & New York & FPL & Rochester Gas \& Electric Corporation & 100.0 \\
\hline Grand Gulf Nuclear Station & Mississippi & FPL & $\begin{array}{l}\text { System Energy Resources, Inc. } \\
\text { South Mississippi Electric Power Association }\end{array}$ & $\begin{array}{l}90.0 \\
10.0\end{array}$ \\
\hline Haddam Neck (Connecticut Yankee) Plant & Connecticut & FPL & Connecticut Yankee Atomic Power Company & 100.0 \\
\hline Harris (Shearon) Nuclear Power Plant & North Carolina & FPL & $\begin{array}{l}\text { Carolina Power \& Light Company } \\
\text { North Carolina Eastern Municipal Power Agency }\end{array}$ & $\begin{array}{l}83.83 \\
16.17\end{array}$ \\
\hline Hatch (Edwin I.) Nuclear Plant, Units $1 \& 2$ & Georgia & FPL & $\begin{array}{l}\text { Georgia Power Company } \\
\text { Oglethorpe Power Corporation } \\
\text { Municipal Electric Authority of Georgia } \\
\text { Dalton Water \& Light Sinking Fund Commission }\end{array}$ & $\begin{array}{r}50.1 \\
30.0 \\
17.7 \\
2.2\end{array}$ \\
\hline Hope Creek Nuclear Generating Station & New Jersey & FPL & $\begin{array}{l}\text { Public Service Electric \& Gas Company } \\
\text { Atlantic City Electric Company }\end{array}$ & $\begin{array}{r}95.0 \\
5.0\end{array}$ \\
\hline
\end{tabular}




\begin{tabular}{cccc}
\hline & Operating & Ownership \\
Plant name & Location & status & Ownership \\
\hline
\end{tabular}

Humboldt Bay Power Plant, Unit 3

Indian Point, Unit 1

Indian Point, Unit 2

Indian Point, Unit 3

Kewaunee Nuclear Power Plant

LaCrosse (Genoa) Nuclear Generating Station

$\infty$

\section{LaSalle County Station, Units $1 \& 2$}

Limerick Generating Station, Units $1 \& 2$

Maine Yankee Atomic Power Station

McGuire (William B.) Nuclear Station, Units 1 \& 2

Millstone Nuclear Power Station, Units 1 \& 2

Millstone Nuclear Power Station, Unit 3
California
New York
New York
New York
Wisconsin

Wisconsin

Illinois

Pennsylvania

Maine

North Carolina

Connecticut

Connecticut
POL Pacific Gas \& Electric Company

POL Consolidated Edison Company of New York

FPL Consolidated Edison Company of New York

FPL New York Power Authority

FPL Wisconsin Public Service Corporation

Wisconsin Power \& Light Company

Madison Gas \& Electric Company

POL Dairyland Power Cooperative

FPL Commonwealth Edison Company

FPL PECO Energy Company

FPL Maine Yankee Atomic Power Company

FPL Duke Power Company

FPL Connecticut Light \& Power Company Western Massachusetts Electric Company

FPL Connecticut Light \& Power Company

Western Massachusetts Electric Company

New England Power Company

Massachusetts Municipal Wholesale Electric

Company

Montaup Electric Company

United Illuminating Company

Public Service Company of New Hampshire
100.0

100.0

100.0

100.0

41.2

41.0

17.8

100.0

100.0

100.0

100.0

100.0

81.0

19.0

52.9330

12.2385

12.2050

4.7990

4.0090

3.6850

2.8475 
Table 1 (continued)

\begin{tabular}{|c|c|c|c|c|}
\hline Plant name & Location & $\begin{array}{l}\text { Operating } \\
\text { status* }\end{array}$ & Ownership & $\begin{array}{l}\text { Ownership } \\
\text { percentage }\end{array}$ \\
\hline \multirow[t]{7}{*}{ Millstone Nuclear Power Station, Unit 3 (continued) } & & & Central Maine Power Company & 2.5000 \\
\hline & & & Central Vermont Public Service Corporation & 1.7303 \\
\hline & & & Chicopee Electric Light Department & 1.3500 \\
\hline & & & $\begin{array}{l}\text { Connecticut Municipal Electric Energy Cooperative, } \\
\text { Inc. }\end{array}$ & 1.0870 \\
\hline & & & $\begin{array}{l}\text { Vermont Electric Generation \& Transmission } \\
\text { Cooperative, Inc. }\end{array}$ & 0.3500 \\
\hline & & & Fitchburg Gas \& Electric Company & 0.2170 \\
\hline & & & Lyndonville (Village of) Electric Department & 0.0487 \\
\hline Monticello Nuclear Generating Plant & Minnesota & FPL & Northern States Power Company & 100.0 \\
\hline Nine Mile Point Nuclear Station, Unit 1 & New York & FPL & Niagara Mohawk Power Corporation & 100.0 \\
\hline \multirow[t]{5}{*}{ Nine Mile Point Nuclear Station, Unit 2} & New York & FPL & Niagara Mohawk Power Corporation & 41.0 \\
\hline & & & Long Island Lighting Company & 18.0 \\
\hline & & & New York State Electric \& Gas Corporation & 18.0 \\
\hline & & & Rochester Gas \& Electric Corporation & 14.0 \\
\hline & & & Central Hudson Gas \& Electric Corporation & 9.0 \\
\hline \multirow[t]{2}{*}{ North Anna Power Station, Units $1 \& 2$} & Virginia & FPL & Virginia Electric and Power Company & 88.4 \\
\hline & & & Old Dominion Electric Cooperative & 11.6 \\
\hline Oconee Nuclear Station, Units 1-3 & South Carolina & FPL & Duke Power Company & 100.0 \\
\hline Oyster Creek Nuclear Power Plant & New Jersey & FPL & Jersey Central Power \& Light Company & 100.0 \\
\hline Palisades Nuclear Plant & Michigan & FPL & Consumers Power Company & 100.0 \\
\hline
\end{tabular}


Table 1 (continued)

\begin{tabular}{cccc}
\hline & Ownership \\
Plant name & Location & $\begin{array}{c}\text { Operating } \\
\text { status* }^{*}\end{array}$ & Ownership \\
\hline
\end{tabular}

Palo Verde Nuclear Generating Station, Units 1-3 Arizona

Pathfinder Atomic Plant

Peach Bottom Atomic Power Station, Unit 1

Peach Bottom Atomic Power Station, Units 2 \& 3

$\overrightarrow{0}$

Perry Nuclear Power Plant

Pilgrim Station

Point Beach Nuclear Plant, Units 1 \& 2

Prairie Island Nuclear Plant, Units 1 \& 2

Quad Cities Station, Units 1 \& 2

FPL Arizona Public Service Company

Salt River Project Agricultural Improvement \& Power

District

EI Paso Electric Company

Southern California Edison Company

Public Service Company of New Mexico

Southern California Public Power Authority

Los Angeles Department of Water \& Power

South Dakota

Pennsylvania

Pennsylvania

Ohio

Massachusetts

Wisconsin

Minnesota

Illinois
P30 Northern States Power Company

POL PECO Energy Company

FPL PECO Energy Company

Public Service Electric \& Gas Company

Delmarva Power \& Light Company

Atlantic City Electric Company

FPL Cleveland Electric Illuminating Company

Ohio Edison Company

Toledo Edison Company

Duquesne Light Company

Pennsylvania Power Company

FPL Boston Edison Company

FPL Wisconsin Electric Power Company

FPL Northern States Power Company

FPL Commonwealth Edison Company MidAmerican Energy Company
29.10

17.49

15.80

15.80

10.20

5.91

5.70

100.0

100.0

42.49

42.49

7.51

7.51

31.11

30.00

19.91

13.74

5.24

100.0

100.0

100.0

75.0 25.0 
Table 1 (continued)

\begin{tabular}{|c|c|c|c|c|}
\hline Plant name & Location & $\begin{array}{l}\text { Operating } \\
\text { status }\end{array}$ & Ownership & $\begin{array}{l}\text { Ownership } \\
\text { percentage }\end{array}$ \\
\hline Rancho Seco Nuclear Generating Station & Califomia & POL & Sacramento Municipal Utility District & 100.0 \\
\hline River Bend Station & Louisiana & FPL & $\begin{array}{l}\text { Entergy Gulf States, Inc. } \\
\text { Cajun Electric Power Cooperative, Inc. }\end{array}$ & $\begin{array}{l}70.0 \\
30.0\end{array}$ \\
\hline Robinson (H. B.) Plant, Unit 2 & South Carolina & FPL & Carolina Power \& Light Company & 100.0 \\
\hline Salem Nuclear Generating Station, Units 1 \& 2 & New Jersey & FPL & $\begin{array}{l}\text { PECO Energy Company } \\
\text { Public Service Electric \& Gas Company } \\
\text { Atlantic City Electric Company } \\
\text { Delmarva Power \& Light Company }\end{array}$ & $\begin{array}{r}42.59 \\
42.59 \\
7.41 \\
7.41\end{array}$ \\
\hline San Onofre Nuclear Generating Station, Unit 1 & California & POL & $\begin{array}{l}\text { Southern California Edison Company } \\
\text { San Diego Gas \& Electric Company }\end{array}$ & $\begin{array}{l}80.0 \\
20.0\end{array}$ \\
\hline San Onofre Nuclear Generating Station, Units 2 \& 3 & California & FPL & $\begin{array}{l}\text { Southern California Edison Company } \\
\text { San Diego Gas \& Electric Company } \\
\text { Anaheim Public Utilities Department } \\
\text { Riverside Utilities Department }\end{array}$ & $\begin{array}{r}75.05 \\
20.00 \\
3.16 \\
1.79\end{array}$ \\
\hline Saxton Nuclear Experimental Reactor Project & Pennsylvania & POL & Saxton Nuclear Experimental Corporation & 100.0 \\
\hline Seabrook Nuclear Power Station & New Hampshire & FPL & $\begin{array}{l}\text { North Atlantic Energy Corporation } \\
\text { United Illuminating Company } \\
\text { Great Bay Power Corporation } \\
\text { Massachusetts Municipal Wholesale Electric } \\
\text { Company }\end{array}$ & $\begin{array}{l}35.98201 \\
17.50000 \\
12.13240 \\
11.59340\end{array}$ \\
\hline & & & $\begin{array}{l}\text { New England Power Company } \\
\text { Connecticut Light \& Power Company } \\
\text { Canal Electric Company } \\
\text { Montaup Electric Company } \\
\text { New Hampshire Electric Cooperative, Inc. } \\
\text { Taunton Municipal Light Plant } \\
\text { Hudson Light \& Power Department }\end{array}$ & $\begin{array}{l}9.95766 \\
4.05985 \\
3.52317 \\
2.89989 \\
2.17391 \\
0.10034 \\
0.07737\end{array}$ \\
\hline
\end{tabular}


Table 1 (continued)

\begin{tabular}{cccc}
\hline & Ownership & Operating \\
Plant name & Location & Status & Ownership
\end{tabular}

Sequoyah Nuclear Plant, Units 1 \& 2

Shoreham Nuclear Power Station

South Texas Project, Units 1 \& 2

St. Lucie Plant, Unit 2

$\overrightarrow{\mathrm{N}}$

Summer (Virgil C.) Nuclear Station

Surry Power Station, Units $1 \& 2$

Susquehanna Steam Electric Station, Units $1 \& 2$

Three Mile Island Nuclear Station, Unit 1

Three Mile Island Nuclear Station, Unit 2

Trojan Nuclear Plant
Tennessee

New York

Texas

Florida

Florida

South Carolina

Virginia

Pennsyivania

Pennsylvania

Pennsylvania

Oregon
FPL Tennessee Valley Authority

100.0

100.0

30.8

28.0

25.2

Santonio City Public Service Board

Central Power \& Light

Austin Electric Department

16.0

100.0

85.10449

8.80600

6.08951

Florida Municipal Power Agency

Orlando Utilities Commission

66.667

33.333

100.0

90.0

10.0

50.0

25.0

25.0

Jersey Central Power \& Light Company

Pennsylvania Electric Company

50.0

25.0

25.0

Jersey Central Power \& Light Company

Pennsylvania Electric Company 
Table 1 (continued)

\begin{tabular}{ccccc}
\hline Plant name & Location & $\begin{array}{c}\text { Operating } \\
\text { status* }^{*}\end{array}$ & Ownership & Ownership \\
percentage
\end{tabular}

Turkey Point Station, Unit $3 \& 4$

Vallecitos Boiling Water Reactor

Vermont Yankee Nuclear Power Station

Vogtle (Alvin W.) Nuclear Plant, Units 1 \& 2

Waterford Generating Station, Unit 3

Watts Bar Nuclear Plant, Unit 1

$\vec{\omega}$

Watts Bar Nuclear Plant, Unit 2

Wolf Creek Generating Station

WPPSS Nuclear Project, Unit 1

WPPSS Nuclear Project, Unit 2

WPPSS Nuclear Project, Unit 3

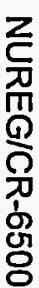

Florida

California

Vermont

Georgia

\section{Louisiana}

Tennessee

Tennessee

Kansas

Washington

Washington

Washington
FPL Florida Power \& Light Company

POL General Electric Company

FPL Vermont Yankee Nuclear Power Corporation

FPL Georgia Power Company

Oglethorpe Power Corporation

Municipal Electric Authority of Georgia

Dalton Water \& Light Sinking Fund Commission

FPL Entergy Louisiana, Inc.

FPL Tennessee Valley Authority

$\mathrm{CDH}$ Tennessee Valley Authority

FPL Kansas Gas \& Electric Company

Kansas City Power \& Light Company

Kansas Electric Power Cooperative, Inc.

$\mathrm{CDH}$ Washington Public Power Supply System

FPL Washington Public Power Supply System

$\mathrm{CDH}$ Washington Public Power Supply System Pacific Power \& Light Company

Portland General Electric Company

Puget Sound Power \& Light Company

Washington Water Power Company
100.0

100.0

100.0

45.7

30.0

22.7

1.6

100.0

100.0

100.0

47.0

47.0

6.0

100.0

100.0

70.0

10.0

10.0

5.0

5.0 


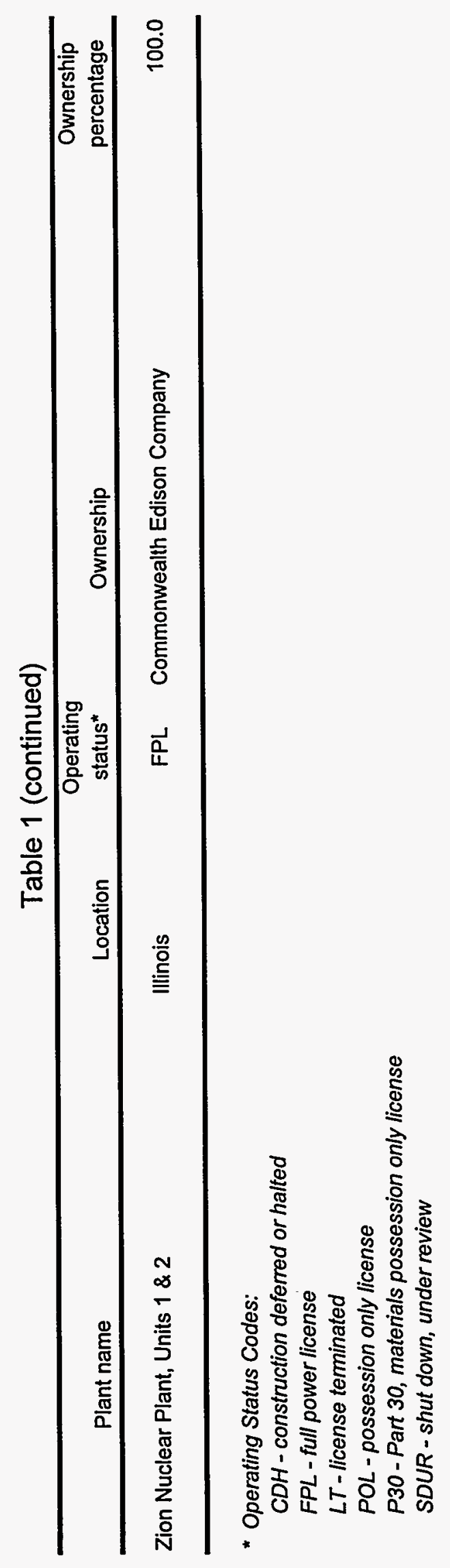

NUREG/CR-6500 


\section{NUCLEAR POWER PLANT PERCENTAGE OWNERSHIP ORDERED BY UTILITY NAME}



Table 2. Nuclear power plant percentage ownership by utility

\begin{tabular}{|c|c|c|}
\hline Utility & Plant name & $\begin{array}{l}\text { Ownership } \\
\text { percentage }\end{array}$ \\
\hline Alabama Power Company & Farley (Joseph M.) Nuclear Plant, Units 1 \& 2 & 100.0 \\
\hline Alachua (City of) Electric Department & Crystal River Nuclear Plant, Unit 3 & 0.0779 \\
\hline Allegheny Electric Cooperative, Inc. & Susquehanna Steam Electric Station, Units 1 \& 2 & 10.0 \\
\hline Anaheim Public Utilities Department & San Onofre Nuclear Generating Station, Units 2 \& 3 & 3.16 \\
\hline Arizona Public Service Company & Palo Verde Nuclear Generating Station, Units 1-3 & 29.1 \\
\hline Atlantic City Electric Company & $\begin{array}{l}\text { Hope Creek Nuclear Generating Station } \\
\text { Peach Bottom Atomic Power Station, Units } 2 \text { \& } 3 \\
\text { Salem Nuclear Generating Station, Units } 1 \& 2\end{array}$ & $\begin{array}{l}5.00 \\
7.51 \\
7.41\end{array}$ \\
\hline Austin Electric Department & South Texas Project, Units 1 \& 2 & 16.0 \\
\hline Baltimore Gas \& Electric Company & Calvert Cliffs Nuclear Power Plant, Units 1 \& 2 & 100.0 \\
\hline Boston Edison Company & Pilgrim Station & 100.0 \\
\hline Bushnell (City of) Utility Department & Crystal River Nuclear Plant, Unit 3 & 0.0388 \\
\hline Cajun Electric Power Cooperative, Inc. & River Bend Station & 30.0 \\
\hline Canal Electric Company & Seabrook Nuclear Power Station & 3.52317 \\
\hline Carolina Power \& Light Company & $\begin{array}{l}\text { Brunswick Steam Electric Plant, Units } 1 \& 2 \\
\text { Harris (Shearon) Nuclear Power Plant } \\
\text { Robinson (H. B.) Plant, Unit } 2\end{array}$ & $\begin{array}{r}81.67 \\
83.83 \\
100.00\end{array}$ \\
\hline Central Hudson Gas \& Electric Corporation & Nine Mile Point Nuclear Station, Unit 2 & 9.0 \\
\hline Central lowa Power Cooperative & Arnold (Duane) Energy Center & 20.0 \\
\hline Central Maine Power Company & Millstone Nuclear Power Station, Unit 3 & 2.5 \\
\hline
\end{tabular}


Table 2 (continued)

\begin{tabular}{|c|c|c|}
\hline Utility & Plant name & $\begin{array}{l}\text { Ownership } \\
\text { percentage }\end{array}$ \\
\hline Central Power \& Light & South Texas Project, Units 1 \& 2 & 25.2 \\
\hline Central Vermont Public Service Corporation & Millstone Nuclear Power Station, Unit 3 & 1.7303 \\
\hline Chicopee Electric Light Department & Millstone Nuclear Power Station, Unit 3 & 1.35 \\
\hline Cleveland Electric Illuminating Company & $\begin{array}{l}\text { Beaver Valley Power Station, Unit } 2 \\
\text { Davis-Besse Nuclear Power Station } \\
\text { Perry Nuclear Power Plant }\end{array}$ & $\begin{array}{l}24.47 \\
51.38 \\
31.11\end{array}$ \\
\hline Commonwealth Edison Company & $\begin{array}{l}\text { Braidwood Station, Units } 1 \text { \& } 2 \\
\text { Byron Station, Units } 1 \text { \& } 2 \\
\text { Dresden Nuclear Power Station, Unit } 1 \\
\text { Dresden Nuclear Power Station, Units } 2 \text { \& } 3 \\
\text { LaSalle County Station, Units } 1 \text { \& } 2 \\
\text { Quad Cities Station, Units } 1 \text { \& } 2 \\
\text { Zion Nuclear Plant, Units } 1 \text { \& } 2\end{array}$ & $\begin{array}{r}100.0 \\
100.0 \\
100.0 \\
100.0 \\
100.0 \\
75.0 \\
100.0\end{array}$ \\
\hline Connecticut Light \& Power Company & $\begin{array}{l}\text { Millstone Nuclear Power Station, Units } 1 \& 2 \\
\text { Millstone Nuclear Power Station, Unit } 3 \\
\text { Seabrook Nuclear Power Station }\end{array}$ & $\begin{array}{r}81.00000 \\
52.93300 \\
4.05985\end{array}$ \\
\hline Connecticut Municipal Electric Energy Cooperative, Inc. & Millstone Nuclear Power Station, Unit 3 & 1.087 \\
\hline Connecticut Yankee Atomic Power Company & Haddam Neck (Connecticut Yankee) Plant & 100.0 \\
\hline Consolidated Edison Company of New York & $\begin{array}{l}\text { Indian Point, Unit } 1 \\
\text { Indian Point, Unit } 2\end{array}$ & $\begin{array}{l}100.0 \\
100.0\end{array}$ \\
\hline Consumers Power Company & $\begin{array}{l}\text { Big Rock Point Nuclear Plant } \\
\text { Palisades Nuclear Plant }\end{array}$ & $\begin{array}{l}100.0 \\
100.0\end{array}$ \\
\hline Corn Belt Power Cooperative & Arnold (Duane) Energy Center & 10.0 \\
\hline Dairyland Power Cooperative & LaCrosse (Genoa) Nuclear Generating Station & 100.0 \\
\hline
\end{tabular}


Table 2 (continued)

\begin{tabular}{|c|c|c|}
\hline Utility & Plant name & $\begin{array}{l}\text { Ownership } \\
\text { percentage }\end{array}$ \\
\hline Dalton Water \& Light Sinking Fund Commission & $\begin{array}{l}\text { Hatch (Edwin l.) Nuclear Plant, Units } 1 \& 2 \\
\text { Vogtle (Alvin W.) Nuclear Plant, Units } 1 \& 2\end{array}$ & $\begin{array}{l}2.2 \\
1.6\end{array}$ \\
\hline Delmarva Power \& Light Company & $\begin{array}{l}\text { Peach Bottom Atomic Power Station, Units } 2 \& 3 \\
\text { Salem Nuclear Generating Station, Units } 1 \& 2\end{array}$ & $\begin{array}{l}7.51 \\
7.41\end{array}$ \\
\hline Detroit Edison Company & Fermi (Enrico) Atomic Power Plant, Unit 2 & 100.0 \\
\hline Duke Power Company & $\begin{array}{l}\text { Catawba Nuclear Station, Unit } 1 \\
\text { McGuire (William B.) Nuclear Station, Units } 1 \text { \& } 2 \\
\text { Oconee Nuclear Station, Units } 1-3\end{array}$ & $\begin{array}{r}25.0 \\
100.0 \\
100.0\end{array}$ \\
\hline Duquesne Light Company & $\begin{array}{l}\text { Beaver Valley Power Station, Unit } 1 \\
\text { Beaver Valley Power Station, Unit } 2 \\
\text { Perry Nuclear Power Plant }\end{array}$ & $\begin{array}{l}47.50 \\
13.74 \\
13.74\end{array}$ \\
\hline El Paso Electric Company & Palo Verde Nuclear Generating Station, Units 1-3 & 15.8 \\
\hline Entergy Arkansas, Inc. & Arkansas Nuclear One, Units $1 \& 2$ & 100.0 \\
\hline Entergy Gulf States, Inc. & River Bend Station & 70.0 \\
\hline Entergy Louisiana, Inc. & Waterford Generating Station, Unit 3 & 100.0 \\
\hline Eugene Water \& Electric Board & Trojan Nuclear Plant & 30.0 \\
\hline Fitchburg Gas \& Electric Company & Millstone Nuclear Power Station, Unit 3 & 0.217 \\
\hline Florida Municipal Power Agency & St. Lucie Plant, Unit 2 & 8.806 \\
\hline Florida Power \& Light Company & $\begin{array}{l}\text { St. Lucie Plant, Unit } 1 \\
\text { St. Lucie Plant, Unit } 2 \\
\text { Turkey Point Station, Unit } 3 \text { \& } 4\end{array}$ & $\begin{array}{r}100.00000 \\
85.10449 \\
100.00000\end{array}$ \\
\hline Fiorida Power Corporation & Crystal River Nuclear Plant, Unit 3 & 90.4473 \\
\hline
\end{tabular}


Table 2 (continued)

\begin{tabular}{|c|c|c|}
\hline Utility & Plant name & $\begin{array}{l}\text { Ownership } \\
\text { percentage }\end{array}$ \\
\hline Gainesville (City of) Regional Utilities & Crystal River Nuclear Plant, Unit 3 & 1.4079 \\
\hline General Electric Company & Vallecitos Boiling Water Reactor & 100.0 \\
\hline Georgia Power Company & $\begin{array}{l}\text { Hatch (Edwin I.) Nuclear Plant, Units } 1 \& 2 \\
\text { Vogtle (Alvin W.) Nuclear Plant, Units } 1 \& 22\end{array}$ & $\begin{array}{l}50.1 \\
45.7\end{array}$ \\
\hline Great Bay Power Corporation & Seabrook Nuclear Power Station & 12.1324 \\
\hline Houston Lighting \& Power & South Texas Project, Units 1 \& 2 & 30.8 \\
\hline Hudson Light \& Power Department & Seabrook Nuclear Power Station & 0.07737 \\
\hline IES Utilities, Inc. & Arnold (Duane) Energy Center & 70.0 \\
\hline Illinois Power Company & Clinton Power Station & 86.79 \\
\hline Indiana Michigan Power Company & Cook (Donald C.) Nuclear Power Plant, Units 1 \& 2 & 100.0 \\
\hline Jersey Central Power \& Light Company & $\begin{array}{l}\text { Oyster Creek Nuclear Power Plant } \\
\text { Three Mile Island Nuclear Station, Unit } 1 \\
\text { Three Mile Island Nuclear Station, Unit } 2\end{array}$ & $\begin{array}{r}100.0 \\
25.0 \\
25.0\end{array}$ \\
\hline Kansas City Power \& Light Company & Wolf Creek Generating Station & 47.0 \\
\hline Kansas Electric Power Cooperative, Inc. & Wolf Creek Generating Station & 6.0 \\
\hline Kansas Gas \& Electric Company & Wolf Creek Generating Station & 47.0 \\
\hline Kissimee (City of) Utilities & Crystal River Nuclear Plant, Unit 3 & 0.6754 \\
\hline Leesburg (City of) Municipal Electric Department & Crystal River Nuclear Plant, Unit 3 & 0.8244 \\
\hline Long Island Lighting Company & Nine Mile Point Nuclear Station, Unit 2 & 18.0 \\
\hline Long Island Power Authority & Shoreham Nuclear Power Station & 100.0 \\
\hline
\end{tabular}


Table 2 (continued)

\begin{tabular}{|c|c|c|}
\hline Utility & Plant name & $\begin{array}{l}\text { Ownership } \\
\text { percentage }\end{array}$ \\
\hline Los Angeles Department of Water \& Power & Palo Verde Nuclear Generating Station, Units 1-3 & 5.7 \\
\hline Lyndonville (Village of) Electric Department & Millstone Nuclear Power Station, Unit 3 & 0.0487 \\
\hline Madison Gas \& Electric Company & Kewaunee Nuclear Power Plant & 17.8 \\
\hline Maine Yankee Atomic Power Company & Maine Yankee Atomic Power Station & 100.0 \\
\hline Massachusetts Municipal Wholesale Electric Company & $\begin{array}{l}\text { Millstone Nuclear Power Station, Unit } 3 \\
\text { Seabrook Nuclear Power Station }\end{array}$ & $\begin{array}{r}4.7990 \\
11.5934\end{array}$ \\
\hline Metropolitan Edison Company & $\begin{array}{l}\text { Three Mile Island Nuclear Station, Unit } 1 \\
\text { Three Mile Island Nuclear Station, Unit } 2\end{array}$ & $\begin{array}{l}50.0 \\
50.0\end{array}$ \\
\hline MidAmerican Energy Company & Quad Cities Station, Units $1 \& 2$ & 25.0 \\
\hline Montaup Electric Company & $\begin{array}{l}\text { Millstone Nuclear Power Station, Unit } 3 \\
\text { Seabrook Nuclear Power Station }\end{array}$ & $\begin{array}{l}4.00900 \\
2.89989\end{array}$ \\
\hline Municipal Electric Authority of Georgia & $\begin{array}{l}\text { Hatch (Edwin I.) Nuclear Plant, Units } 1 \& 2 \\
\text { Vogtle (Alvin W.) Nuclear Plant, Units } 1 \& 2\end{array}$ & $\begin{array}{l}17.7 \\
22.7\end{array}$ \\
\hline Nebraska Public Power District & Cooper Nuclear Station & 100.0 \\
\hline New England Power Company & $\begin{array}{l}\text { Millstone Nuclear Power Station, Unit } 3 \\
\text { Seabrook Nuclear Power Station }\end{array}$ & $\begin{array}{r}12.20500 \\
9.95766\end{array}$ \\
\hline New Hampshire Electric Cooperative, Inc. & Seabrook Nuclear Power Station & 2.17391 \\
\hline New Smyrna Beach Utilities Commission & Crystal River Nuclear Plant, Unit 3 & 0.5608 \\
\hline New York Power Authority & $\begin{array}{l}\text { Fitzpatrick (James A.) Nuclear Power Plant } \\
\text { Indian Point, Unit } 3\end{array}$ & $\begin{array}{l}100.0 \\
100.0\end{array}$ \\
\hline New York State Electric \& Gas Corporation & Nine Mile Point Nuclear Station, Unit 2 & 18.0 \\
\hline
\end{tabular}


Table 2 (continued)

\begin{tabular}{|c|c|c|}
\hline Utility & Plant name & $\begin{array}{r}\text { Ownership } \\
\text { percentage }\end{array}$ \\
\hline Niagara Mohawk Power Corporation & $\begin{array}{l}\text { Nine Mile Point Nuclear Station, Unit } 1 \\
\text { Nine Mile Point Nuclear Station, Unit } 2\end{array}$ & $\begin{array}{r}100.0 \\
41.0\end{array}$ \\
\hline North Atlantic Energy Corporation & Seabrook Nuclear Power Station & 35.98201 \\
\hline North Carolina Eastern Municipal Power Agency & $\begin{array}{l}\text { Brunswick Steam Electric Plant, Units } 1 \text { \& } 2 \\
\text { Catawba Nuclear Station, Unit } 2 \\
\text { Harris (Shearon) Nuclear Power Plant }\end{array}$ & $\begin{array}{l}18.33 \\
75.00 \\
16.17\end{array}$ \\
\hline North Carolina Electric Membership Corporation & Catawba Nuclear Station, Unit 1 & 56.25 \\
\hline Northern States Power Company & $\begin{array}{l}\text { Monticello Nuclear Generating Plant } \\
\text { Pathfinder Atomic Plant } \\
\text { Prairie Island Nuclear Plant, Units } 1 \text { \& } 2\end{array}$ & $\begin{array}{l}100.0 \\
100.0 \\
100.0\end{array}$ \\
\hline Ocala (City of) Utilities Division & Crystal River Nuclear Plant, Unit 3 & 1.3333 \\
\hline Oglethorpe Power Corporation & $\begin{array}{l}\text { Hatch (Edwin I.) Nuclear Plant, Units } 1 \& 2 \\
\text { Vogtle (Alvin W.) Nuclear Plant, Units } 1 \& 2\end{array}$ & $\begin{array}{l}30.0 \\
30.0\end{array}$ \\
\hline Ohio Edison Company & $\begin{array}{l}\text { Beaver Valley Power Station, Unit } 1 \\
\text { Beaver Valley Power Station, Unit } 2 \\
\text { Perry Nuclear Power Plant }\end{array}$ & $\begin{array}{l}35.00 \\
41.88 \\
30.00\end{array}$ \\
\hline Old Dominion Electric Cooperative & North Anna Power Station, Units 1 \& 2 & 11.6 \\
\hline Omaha Public Power District & Fort Calhoun Station & 100.0 \\
\hline Orlando Utilities Commission & $\begin{array}{l}\text { Crystal River Nuclear Plant, Unit } 3 \\
\text { St. Lucie Plant, Unit } 2\end{array}$ & $\begin{array}{l}1.60150 \\
6.08951\end{array}$ \\
\hline Pacific Gas \& Electric Company & $\begin{array}{l}\text { Diablo Canyon Nuclear Power Plant, Units } 1 \text { \& } 2 \\
\text { Humboldt Bay Power Plant, Unit } 3\end{array}$ & $\begin{array}{l}100.0 \\
100.0\end{array}$ \\
\hline Pacific Power \& Light Company & $\begin{array}{l}\text { Trojan Nuclear Plant } \\
\text { WPPSS Nuclear Project, Unit } 3\end{array}$ & $\begin{array}{r}2.5 \\
10.0 \\
\end{array}$ \\
\hline
\end{tabular}


Table 2 (continued)

\begin{tabular}{|c|c|c|}
\hline Utility & Plant name & $\begin{array}{l}\text { Ownership } \\
\text { percentage }\end{array}$ \\
\hline PECO Energy Company & $\begin{array}{l}\text { Limerick Generating Station, Units } 1 \text { \& } 2 \\
\text { Peach Bottom Atomic Power Station, Unit } 1 \\
\text { Peach Bottom Atomic Power Station, Units } 2 \text { \& } 3 \\
\text { Salem Nuclear Generating Station, Units } 1 \text { \& } 2\end{array}$ & $\begin{array}{r}100.00 \\
100.00 \\
42.49 \\
42.59\end{array}$ \\
\hline Pennsylvania Electric Company & $\begin{array}{l}\text { Three Mile Island Nuclear Station, Unit } 1 \\
\text { Three Mile Island Nuclear Station, Unit } 2\end{array}$ & $\begin{array}{l}25.0 \\
25.0\end{array}$ \\
\hline Pennsylvania Power \& Light Company & Susquehanna Steam Electric Station, Units 1 \& 2 & 90.0 \\
\hline Pennsylvania Power Company & $\begin{array}{l}\text { Beaver Valley Power Station, Unit } 1 \\
\text { Perry Nuclear Power Plant }\end{array}$ & $\begin{array}{r}17.50 \\
5.24\end{array}$ \\
\hline Piedmont Municipal Power Agency & Catawba Nuclear Station, Unit 2 & 25.0 \\
\hline Portland General Electric Company & $\begin{array}{l}\text { Trojan Nuclear Plant } \\
\text { WPPSS Nuclear Project, Unit } 3\end{array}$ & $\begin{array}{l}67.5 \\
10.0\end{array}$ \\
\hline Power Reactor Development Company & Fermi (Enrico) Atomic Power Plant, Unit 1 & 100.0 \\
\hline Public Service Company of Colorado & Fort St. Vrain Nuclear Generating Station & 100.0 \\
\hline Public Service Company of New Hampshire & Millstone Nuclear Power Station, Unit 3 & 2.8475 \\
\hline Public Service Company of New Mexico & Palo Verde Nuclear Generating Station, Units 1-3 & 10.2 \\
\hline Public Service Electric \& Gas Company & $\begin{array}{l}\text { Hope Creek Nuclear Generating Station } \\
\text { Peach Bottom Atomic Power Station, Units } 2 \& 3 \\
\text { Salem Nuclear Generating Station, Units } 1 \text { \& } 2\end{array}$ & $\begin{array}{l}95.00 \\
42.49 \\
42.59\end{array}$ \\
\hline Puget Sound Power \& Light Company & WPPSS Nuclear Project, Unit 3 & 5.0 \\
\hline Riverside Utilities Department & San Onofre Nuclear Generating Station, Units 2 \& 3 & 1.79 \\
\hline Rochester Gas \& Electric Corporation & $\begin{array}{l}\text { Ginna (Robert E.) Nuclear Power Plant } \\
\text { Nine Mile Point Nuclear Station, Unit } 2\end{array}$ & $\begin{array}{r}100.0 \\
14.0\end{array}$ \\
\hline
\end{tabular}


Table 2 (continued)

\begin{tabular}{|c|c|c|}
\hline Utility & Plant name & $\begin{array}{l}\text { Ownership } \\
\text { percentage }\end{array}$ \\
\hline Sacramento Municipal Utility District & Rancho Seco Nuclear Generating Station & 100.0 \\
\hline Salt River Project Agricultural Improvement \& Power District & Palo Verde Nuclear Generating Station, Units 1-3 & 17.49 \\
\hline Saluda River Electric Cooperative & Catawba Nuclear Station, Unit 1 & 18.75 \\
\hline San Antonio City Public Service Board & South Texas Project, Units 1 \& 2 & 28.0 \\
\hline San Diego Gas \& Electric Company & $\begin{array}{l}\text { San Onofre Nuclear Generating Station, Unit } 1 \\
\text { San Onofre Nuclear Generating Station, Units } 2 \text { \& } 3\end{array}$ & $\begin{array}{l}20.0 \\
20.0\end{array}$ \\
\hline Saxton Nuclear Experimental Corporation & Saxton Nuclear Experimental Reactor Project & 100.0 \\
\hline Seminole Electric Cooperative, Inc. & Crystal River Nuclear Plant, Unit 3 & 1.6994 \\
\hline South Carolina Electric \& Gas Company & Summer (Virgil C.) Nuclear Station & 66.667 \\
\hline South Carolina Public Service Authority & Summer (Virgil C.) Nuclear Station & 33.333 \\
\hline South Mississippi Electric Power Association & Grand Gulf Nuclear Station & 10.0 \\
\hline Southern California Edison Company & $\begin{array}{l}\text { Palo Verde Nuclear Generating Station, Units 1-3 } \\
\text { San Onofre Nuclear Generating Station, Unit } 1 \\
\text { San Onofre Nuclear Generating Station, Units } 2 \text { \& } 3\end{array}$ & $\begin{array}{l}15.80 \\
80.00 \\
75.05\end{array}$ \\
\hline Southern California Public Power Authority & Palo Verde Nuclear Generating Station, Units 1-3 & 5.91 \\
\hline Soyland Power Cooperative, Inc. & Clinton Power Station & 13.21 \\
\hline System Energy Resources, Inc. & Grand Gulf Nuclear Station & 90.0 \\
\hline Tallahassee (City of) Electric Department & Crystal River Nuclear Plant, Unit 3 & 1.3333 \\
\hline Taunton Municipal Light Plant & Seabrook Nuclear Power Station & 0.10034 \\
\hline
\end{tabular}


Table 2 (continued)

Utility

Tennessee Valley Authority

Toledo Edison Company

TU Electric Company

Union Electric Company

N

United Illuminating Company

Vermont Electric Generation \& Transmission Cooperative, Inc.

Vermont Yankee Nuclear Power Corporation

Virginia Electric and Power Company

Washington Public Power Supply System

Washington Water Power Company

Western Massachusetts Electric Company

Wisconsin Electric Power Company
Plant name

Ownership

percentage

Bellefonte Nuclear Plant, Units 1 \& 2

Brown's Ferry Nuclear Power Station, Unit 1

100.0

100.0

100.0

100.0

Sequoyah Nuclear Plant, Units 1 \& 2

Watts Bar Nuclear Plant, Unit 1

Watts Bar Nuclear Plant, Unit 2

Beaver Valley Power Station, Unit 2

Davis-Besse Nuclear Power Station

Perry Nuclear Power Plant

19.91

48.62

19.91

Comanche Peak Steam Electric Station, Units 1 \& 2

100.0

Callaway Plant

100.0

Millstone Nuclear Power Station, Unit 3

3.685

Seabrook Nuclear Power Station

17.500

Millstone Nuclear Power Station, Unit 3

0.35

Vermont Yankee Nuclear Power Station $\quad 100.0$

North Anna Power Station, Units 1 \& 2

Surry Power Station, Units 1 \& 2

WPPSS Nuclear Project, Unit 1

WPPSS Nuclear Project, Unit $2 \quad 100.0$

WPPSS Nuclear Project, Unit 3

WPPSS Nuclear Project, Unit $3 \quad 5.0$

Millstone Nuclear Power Station, Units 1 \& $2 \quad 19.0000$

Milistone Nuclear Power Station, Unit $3 \quad 12.2385$

$\begin{array}{lr}\text { Point Beach Nuclear Plant, Units } 1 \text { \& } 2 & 100.0\end{array}$ 
Table 2 (continued)

\begin{tabular}{|c|c|c|}
\hline Utility & Plant name & $\begin{array}{r}\text { Ownership } \\
\text { percentage }\end{array}$ \\
\hline Wisconsin Power \& Light Company & Kewaunee Nuclear Power Plant & 41.0 \\
\hline Wisconsin Public Service Corporation & Kewaunee Nuclear Power Plant & 41.2 \\
\hline Yankee Atomic Electric Company & Yankee Nuclear Power Station & 100.0 \\
\hline
\end{tabular}




\section{UTILITY/COMPANY RELATIONSHIPS ORDERED BY PARENT/HOLDING COMPANY}



Table 3. Utility/company relationships by parent/holding company

\begin{tabular}{|c|c|c|}
\hline Parentholding company & Subsidiary & $\begin{array}{l}\text { Ownership } \\
\text { percentage }\end{array}$ \\
\hline American Electric Power Company, Inc. & Indiana Michigan Power Company & 100.0 \\
\hline Atlantic Energy, Inc. & Atlantic City Electric Company & 100.0 \\
\hline Bangor Hydro-Electric Company & Maine Yankee Atomic Power Company & 7.0 \\
\hline \multirow[t]{2}{*}{ Boston Edison Company } & Connecticut Yankee Atomic Power Company & 9.5 \\
\hline & Yankee Atomic Electric Company & 9.5 \\
\hline Burlington Electric Department & Vermont Yankee Nuclear Power Corporation & 3.6 \\
\hline \multirow[t]{3}{*}{ Cambridge Electric Light Company } & Maine Yankee Atomic Power Company & 4.0 \\
\hline & Vermont Yankee Nuclear Power Corporation & 2.5 \\
\hline & Yankee Atomic Electric Company & 2.0 \\
\hline \multirow[t]{2}{*}{ Centerior Energy Corporation } & Cleveland Electric Illuminating Company & 100.0 \\
\hline & Toledo Edison Company & 100.0 \\
\hline Central and South West Corporation & Central Power \& Light & 100.0 \\
\hline \multirow[t]{4}{*}{ Central Maine Power Company } & Connecticut Yankee Atomic Power Company & 6.0 \\
\hline & Maine Yankee Atomic Power Company & 38.0 \\
\hline & Vermont Yankee Nuclear Power Corporation & 4.0 \\
\hline & Yankee Atomic Electric Company & 9.5 \\
\hline \multirow[t]{4}{*}{ Central Vermont Public Service Corporation } & Connecticut Yankee Atomic Power Company & 2.0 \\
\hline & Maine Yankee Atomic Power Company & 2.0 \\
\hline & Vermont Yankee Nuclear Power Corporation & 31.3 \\
\hline & Yankee Atomic Electric Company & 3.5 \\
\hline CMS Energy Corporation & Consumers Power Company & 100.0 \\
\hline
\end{tabular}


Table 3 (continued)

\begin{tabular}{|c|c|c|}
\hline Parentholding company & Subsidiary & $\begin{array}{r}\text { Ownership } \\
\text { percentage }\end{array}$ \\
\hline \multirow[t]{2}{*}{ Commonwealth Electric Company } & Connecticut Yankee Atomic Power Company & 4.5 \\
\hline & Yankee Atomic Electric Company & 2.5 \\
\hline \multirow[t]{2}{*}{ Commonwealth Energy System } & Cambridge Electric Light Company & 100.0 \\
\hline & Commonwealth Electric Company & 100.0 \\
\hline \multirow[t]{4}{*}{ Connecticut Light \& Power Company } & Connecticut Yankee Atomic Power Company & 34.5 \\
\hline & Maine Yankee Atomic Power Company & 12.0 \\
\hline & Vermont Yankee Nuclear Power Corporation & 9.5 \\
\hline & Yankee Atomic Electric Company & 24.5 \\
\hline Dominion Resources, Inc. & Virginia Electric and Power Company & 100.0 \\
\hline DQE & Duquesne Light Company & 100.0 \\
\hline Eastern Utilities Associates & Montaup Electric Company & 100.0 \\
\hline Edison International & Southern California Edison Company & 100.0 \\
\hline Enova Corporation & San Diego Gas \& Electric Company & 100.0 \\
\hline \multirow[t]{5}{*}{ Entergy Corporation } & Entergy Arkansas, Inc. & 100.0 \\
\hline & Entergy Gulf States, Inc. & 100.0 \\
\hline & Entergy Louisiana, Inc. & 100.0 \\
\hline & Entergy Operations, Inc. & 100.0 \\
\hline & System Energy Resources, Inc. & 100.0 \\
\hline Florida Progress Corporation & Florida Power Corporation & 100.0 \\
\hline FPL Group, Inc. & Florida Power \& Light Company & 100.0 \\
\hline
\end{tabular}


Table 3 (continued)

\begin{tabular}{|c|c|c|}
\hline Parentholding company & Subsidiary & $\begin{array}{l}\text { Ownership } \\
\text { percentage }\end{array}$ \\
\hline \multirow[t]{4}{*}{ GPU, Inc. } & GPU Nuclear Corporation & 100.0 \\
\hline & Jersey Central Power \& Light Company & 100.0 \\
\hline & Metropolitan Edison Company & 100.0 \\
\hline & Pennsylvania Electric Company & 100.0 \\
\hline Green Mountain Power Corporation & Vermont Yankee Nuclear Power Corporation & 17.9 \\
\hline Houston Industries, Inc. & Houston Lighting \& Power & 100.0 \\
\hline IES Industries, Inc. & IES Utilities, Inc. & 100.0 \\
\hline Illinova Corporation & Illinois Power Company & 100.0 \\
\hline IPC Development Company & Interstate Power Company & 100.0 \\
\hline Kansas City Power \& Light Company & Wolf Creek Nuclear Operating Company & 47.0 \\
\hline Kansas Electric Power Cooperative, Inc. & Wolf Creek Nuclear Operating Company & 6.0 \\
\hline Kansas Gas \& Electric Company & Wolf Creek Nuclear Operating Company & 47.0 \\
\hline Lyndonville (Village of) Electric Department & Vermont Yankee Nuclear Power Corporation & 0.6 \\
\hline Maine Public Service Company & Maine Yankee Atomic Power Company & 5.0 \\
\hline \multirow[t]{4}{*}{ Montaup Electric Company } & Connecticut Yankee Atomic Power Company & 4.5 \\
\hline & Maine Yankee Atomic Power Company & 4.0 \\
\hline & Vermont Yankee Nuclear Power Corporation & 2.5 \\
\hline & Yankee Atomic Electric Company & 4.5 \\
\hline New England Electric System & New England Power Company & 100.0 \\
\hline
\end{tabular}


Table 3 (continued)

\begin{tabular}{|c|c|c|}
\hline Parent/holding company & Subsidiary & $\begin{array}{l}\text { Ownership } \\
\text { percentage }\end{array}$ \\
\hline \multirow[t]{4}{*}{ New England Power Company } & Connecticut Yankee Atomic Power Company & 15.0 \\
\hline & Maine Yankee Atomic Power Company & 20.0 \\
\hline & Vermont Yankee Nuclear Power Corporation & 20.0 \\
\hline & Yankee Atomic Electric Company & 30.0 \\
\hline \multirow[t]{5}{*}{ Northeast Utilities } & Connecticut Light \& Power Company & 100.0 \\
\hline & North Atlantic Energy Corporation & 100.0 \\
\hline & North Atlantic Energy Service Corporation & 100.0 \\
\hline & Public Service Company of New Hampshire & 100.0 \\
\hline & Western Massachusetts Electric Company & 100.0 \\
\hline Ohio Edison Company & Pennsylvania Power Company & 100.0 \\
\hline PACIFICORP & Pacific Power \& Light Company & 100.0 \\
\hline Pinnacle West Capital Corporation & Arizona Public Service Company & 100.0 \\
\hline Portland General Corporation & Portland General Electric Company & 100.0 \\
\hline PP\&L Resources, Inc. & Pennsyivania Power \& Light Company & 100.0 \\
\hline \multirow[t]{4}{*}{ Public Service Company of New Hampshire } & Connecticut Yankee Atomic Power Company & 5.0 \\
\hline & Maine Yankee Atomic Power Company & 5.0 \\
\hline & Vermont Yankee Nuclear Power Corporation & 4.0 \\
\hline & Yankee Atomic Electric Company & 7.0 \\
\hline Public Service Enterprise Group, Inc. & Public Service Electric \& Gas Company & 100.0 \\
\hline SCANA Corporation & South Carolina Electric \& Gas Company & 100.0 \\
\hline \multirow[t]{3}{*}{ Southern Company (The) } & Alabama Power Company & 100.0 \\
\hline & Georgia Power Company & 100.0 \\
\hline & Southern Nuclear Operating Company (The) & 100.0 \\
\hline
\end{tabular}


Table 3 (continued)

Parent/holding company

Texas Utilities Company

Unicom Corporation

United Illuminating Company

UNITIL Corporation

Vermont Electric Generation \& Transmission Cooperative, Inc.

Washington Electric Cooperative

Western Massachusetts Electric Company

$\omega$

Western Resources, Inc.

Wisconsin Energy Corporation

WPL Holdings, Inc.
Subsidiary

Ownership

percentage

TU Electric Company

100.0

Commonwealth Edison Company

100.0

Connecticut Yankee Atomic Power Company

Fitchburg Gas \& Electric Company

100.0

Vermont Yankee Nuclear Power Corporation

Vermont Yankee Nuclear Power Corporation

0.6

Connecticut Yankee Atomic Power Company

Maine Yankee Atomic Power Company

Vermont Yankee Nuclear Power Corporation

Yankee Atomic Electric Company

3.0

2.5

7.0

Kansas Gas \& Electric Company

Wisconsin Electric Power Company

100.0

Wisconsin Power \& Light Company

100.0

Wisconsin Public Service Corporation 


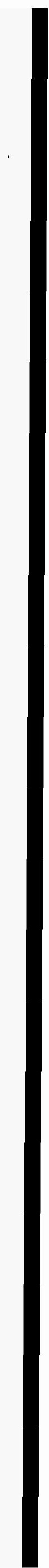




\section{UTILITY/COMPANY RELATIONSHIPS ORDERED BY SUBSIDIARY}



Table 4. Utility/company relationships by subsidiary

\begin{tabular}{|c|c|c|}
\hline Subsidiary & Parent/holding company & $\begin{array}{l}\text { Ownership } \\
\text { percentage }\end{array}$ \\
\hline Alabama Power Company & Southern Company (The) & 100.0 \\
\hline Arizona Public Service Company & Pinnacle West Capital Corporation & 100.0 \\
\hline Atlantic City Electric Company & Atlantic Energy, Inc. & 100.0 \\
\hline Cambridge Electric Light Company & Commonwealth Energy System & 100.0 \\
\hline Central Power \& Light & Central and South West Corporation & 100.0 \\
\hline Cleveland Electric Illuminating Company & Centerior Energy Corporation & 100.0 \\
\hline Commonwealth Edison Company & Unicom Corporation & 100.0 \\
\hline Commonwealth Electric Company & Commonwealth Energy System & 100.0 \\
\hline Connecticut Light \& Power Company & Northeast Utilities & 100.0 \\
\hline \multirow[t]{10}{*}{ Connecticut Yankee Atomic Power Company } & Boston Edison Company & 9.5 \\
\hline & Central Maine Power Company & 6.0 \\
\hline & Central Vermont Public Service Corporation & 2.0 \\
\hline & Commonwealth Electric Company & 4.5 \\
\hline & Connecticut Light \& Power Company & 34.5 \\
\hline & Montaup Electric Company & 4.5 \\
\hline & New England Power Company & 15.0 \\
\hline & Public Service Company of New Hampshire & 5.0 \\
\hline & United Illuminating Company & 9.5 \\
\hline & Western Massachusetts Electric Company & 9.5 \\
\hline Consumers Power Company & CMS Energy Corporation & 100.0 \\
\hline
\end{tabular}


Table 4 (continued)

\begin{tabular}{|c|c|c|}
\hline Subsidiary & Parent/holding company & $\begin{array}{l}\text { Ownership } \\
\text { percentage }\end{array}$ \\
\hline Duquesne Light Company & DQE & 100.0 \\
\hline Entergy Arkansas, Inc. & Entergy Corporation & 100.0 \\
\hline Entergy Gulf States, Inc. & Entergy Corporation & 100.0 \\
\hline Entergy Louisiana, Inc. & Entergy Corporation & 100.0 \\
\hline Entergy Operations, Inc. & Entergy Corporation & 100.0 \\
\hline Fitchburg Gas \& Electric Company & UNITIL Corporation & 100.0 \\
\hline Florida Power \& Light Company & FPL Group, Inc. & 100.0 \\
\hline Florida Power Corporation & Florida Progress Corporation & 100.0 \\
\hline Georgia Power Company & Southern Company (The) & 100.0 \\
\hline GPU Nuclear Corporation & GPU, Inc. & 100.0 \\
\hline Houston Lighting \& Power & Houston Industries, Inc. & 100.0 \\
\hline IES Utilities, Inc. & IES Industries, Inc. & 100.0 \\
\hline Illinois Power Company & Illinova Corporation & 100.0 \\
\hline Indiana Michigan Power Company & American Electric Power Company, Inc. & 100.0 \\
\hline Interstate Power Company & IPC Development Company & 100.0 \\
\hline
\end{tabular}


Table 4 (continued)

\begin{tabular}{|c|c|c|}
\hline Subsidiary & Parent/holding company & $\begin{array}{l}\text { Ownership } \\
\text { percentage }\end{array}$ \\
\hline Jersey Central Power \& Light Company & GPU, Inc. & 100.0 \\
\hline Kansas Gas \& Electric Company & Western Resources, Inc. & 100.0 \\
\hline \multirow[t]{10}{*}{ Maine Yankee Atomic Power Company } & Bangor Hydro-Electric Company & 7.0 \\
\hline & Cambridge Electric Light Company & 4.0 \\
\hline & Central Maine Power Company & 38.0 \\
\hline & Central Vermont Public Service Corporation & 2.0 \\
\hline & Connecticut Light \& Power Company & 12.0 \\
\hline & Maine Public Service Company & 5.0 \\
\hline & Montaup Electric Company & 4.0 \\
\hline & New England Power Company & 20.0 \\
\hline & Public Service Company of New Hampshire & 5.0 \\
\hline & Western Massachusetts Electric Company & 3.0 \\
\hline Metropolitan Edison Company & GPU, Inc. & 100.0 \\
\hline Montaup Electric Company & Eastern Utilities Associates & 100.0 \\
\hline New England Power Company & New England Electric System & 100.0 \\
\hline North Atlantic Energy Corporation & Northeast Utilities & 100.0 \\
\hline North Atlantic Energy Service Corporation & Northeast Utilities & 100.0 \\
\hline Pacific Power \& Light Company & PACIFICORP & 100.0 \\
\hline Pennsylvania Electric Company & GPU, Inc. & 100.0 \\
\hline Pennsylvania Power \& Light Company & PP\&L Resources, Inc. & 100.0 \\
\hline Pennsylvania Power Company & Ohio Edison Company & 100.0 \\
\hline
\end{tabular}




\begin{tabular}{|c|c|c|}
\hline Subsidiary & Parent/holding company & $\begin{array}{l}\text { Ownership } \\
\text { percentage }\end{array}$ \\
\hline Portland General Electric Company & Portland General Corporation & 100.0 \\
\hline Public Service Company of New Hampshire & Northeast Utilities & 100.0 \\
\hline Public Service Electric \& Gas Company & Public Service Enterprise Group, Inc. & 100.0 \\
\hline San Diego Gas \& Electric Company & Enova Corporation & 100.0 \\
\hline South Carolina Electric \& Gas Company & SCANA Corporation & 100.0 \\
\hline Southern California Edison Company & Edison International & 100.0 \\
\hline Southern Nuclear Operating Company (The) & Southern Company (The) & 100.0 \\
\hline System Energy Resources, Inc. & Entergy Corporation & 100.0 \\
\hline Toledo Edison Company & Centerior Energy Corporation & 100.0 \\
\hline TU Electric Company & Texas Utilities Company & 100.0 \\
\hline \multirow[t]{10}{*}{ Vermont Yankee Nuclear Power Corporation } & Burlington Electric Department & 3.6 \\
\hline & Cambridge Electric Light Company & 2.5 \\
\hline & Central Maine Power Company & 4.0 \\
\hline & Central Vermont Public Service Corporation & 31.3 \\
\hline & Connecticut Light \& Power Company & 9.5 \\
\hline & Green Mountain Power Corporation & 17.9 \\
\hline & Lyndonville (Village of) Electric Department & 0.6 \\
\hline & Montaup Electric Company & 2.5 \\
\hline & New England Power Company & 20.0 \\
\hline & Public Service Company of New Hampshire & 4.0 \\
\hline
\end{tabular}


Table 4 (continued)

\begin{tabular}{|c|c|c|}
\hline Subsidiary & Parent/holding company & $\begin{array}{l}\text { Ownership } \\
\text { percentage }\end{array}$ \\
\hline \multirow[t]{3}{*}{ Vermont Yankee Nuclear Power Corp. (continued) } & Vermont Electric Generation \& Transmission Cooperative, Inc. & 1.0 \\
\hline & Washington Electric Cooperative & 0.6 \\
\hline & Western Massachusetts Electric Company & 2.5 \\
\hline Virginia Electric and Power Company & Dominion Resources, Inc. & 100.0 \\
\hline Western Massachusetts Electric Company & Northeast Utilities & 100.0 \\
\hline Wisconsin Electric Power Company & Wisconsin Energy Corporation & 100.0 \\
\hline Wisconsin Power \& Light Company & WPL Holdings, Inc. & 100.0 \\
\hline Wisconsin Public Service Corporation & WPS Resources Corporation & 100.0 \\
\hline \multirow[t]{3}{*}{ Wolf Creek Nuclear Operating Company } & Kansas City Power \& Light Company & 47.0 \\
\hline & Kansas Electric Power Cooperative, Inc. & 6.0 \\
\hline & Kansas Gas \& Electric Company & 47.0 \\
\hline \multirow[t]{10}{*}{ Yankee Atomic Electric Company } & Boston Edison Company & 9.5 \\
\hline & Cambridge Electric Light Company & 2.0 \\
\hline & Central Maine Power Company & 9.5 \\
\hline & Central Vermont Public Service Corporation & 3.5 \\
\hline & Commonwealth Electric Company & 2.5 \\
\hline & Connecticut Light \& Power Company & 24.5 \\
\hline & Montaup Electric Company & 4.5 \\
\hline & New England Power Company & 30.0 \\
\hline & Public Service Company of New Hampshire & 7.0 \\
\hline & Western Massachusetts Electric Company & 7.0 \\
\hline
\end{tabular}





\section{NUCLEAR POWER PLANTS LISTED BY OPERATOR}



Table 5. Nuclear power plants listed by operator

\begin{tabular}{|c|c|}
\hline Operator & Plant name \\
\hline Arizona Public Service Company & Palo Verde Nuclear Generating Station, Units $1-3$ \\
\hline Baltimore Gas \& Electric Company & Calvert Cliffs Nuclear Power Plant, Units 1 \& 2 \\
\hline Boston Edison Company & Pilgrim Station \\
\hline Carolina Power \& Light Company & $\begin{array}{l}\text { Brunswick Steam Electric Plant, Units } 1 \& 2 \\
\text { Harris (Shearon) Nuclear Power Plant } \\
\text { Robinson (H. B.) Plant, Unit } 2\end{array}$ \\
\hline Cleveland Electric Illuminating Company & Perry Nuclear Power Plant \\
\hline Commonwealth Edison Company & $\begin{array}{l}\text { Braidwood Station, Units } 1 \text { \& } 2 \\
\text { Byron Station, Units } 1 \text { \& } 2 \\
\text { Dresden Nuclear Power Station, Unit } 1 \\
\text { Dresden Nuclear Power Station, Units } 2 \text { \& } 3 \\
\text { LaSalle County Station, Units } 1 \text { \& } 2 \\
\text { Quad Cities Station, Units } 1 \text { \& } 2 \\
\text { Zion Nuclear Plant, Units } 1 \text { \& } 2\end{array}$ \\
\hline Consolidated Edison Company of New York & $\begin{array}{l}\text { Indian Point, Unit } 1 \\
\text { Indian Point, Unit } 2\end{array}$ \\
\hline Consumers Power Company & $\begin{array}{l}\text { Big Rock Point Nuclear Plant } \\
\text { Palisades Nuclear Plant }\end{array}$ \\
\hline Dairyland Power Cooperative & LaCrosse (Genoa) Nuclear Generating Station \\
\hline Detroit Edison Company & Fermi (Enrico) Atomic Power Plant, Unit 2 \\
\hline Duke Power Company & $\begin{array}{l}\text { Catawba Nuclear Station, Unit } 1 \\
\text { Catawba Nuclear Station, Unit } 2 \\
\text { McGuire (William B.) Nuclear Station, Units } 1 \& 2 \\
\text { Oconee Nuclear Station, Units } 1-3\end{array}$ \\
\hline Duquesne Light Company & $\begin{array}{l}\text { Beaver Valley Power Station, Unit } 1 \\
\text { Beaver Valley Power Station, Unit } 2\end{array}$ \\
\hline Entergy Operations, Inc. & $\begin{array}{l}\text { Arkansas Nuclear One, Units } 1 \& 2 \\
\text { Grand Gulf Nuclear Station } \\
\text { River Bend Station } \\
\text { Waterford Generating Station, Unit } 3\end{array}$ \\
\hline Florida Power \& Light Company & $\begin{array}{l}\text { St. Lucie Plant, Unit } 1 \\
\text { St. Lucie Plant, Unit } 2 \\
\text { Turkey Point Station, Unit } 3 \text { \& } 4\end{array}$ \\
\hline
\end{tabular}




\begin{tabular}{|c|c|}
\hline Operator & Plant name \\
\hline Florida Power Corporation & Crystal River Nuclear Plant, Unit 3 \\
\hline General Electric Company & Vallecitos Boiling Water Reactor \\
\hline GPU Nuclear Corporation & $\begin{array}{l}\text { Oyster Creek Nuclear Power Plant } \\
\text { Three Mile Island Nuclear Station, Unit } 1 \\
\text { Three Mile Island Nuclear Station, Unit } 2\end{array}$ \\
\hline Houston Lighting \& Power & South Texas Project, Units $1 \& 2$ \\
\hline IES Utilities, Inc. & Arnold (Duane) Energy Center \\
\hline Illinois Power Company & Clinton Power Station \\
\hline Indiana Michigan Power Company & Cook (Donald C.) Nuclear Power Plant, Units $1 \& 2$ \\
\hline Long Island Lighting Company & Shoreham Nuclear Power Station \\
\hline Maine Yankee Atomic Power Company & Maine Yankee Atomic Power Station \\
\hline Nebraska Public Power District & Cooper Nuclear Station \\
\hline New York Power Authority & $\begin{array}{l}\text { Fitzpatrick (James A.) Nuclear Power Plant } \\
\text { Indian Point, Unit } 3\end{array}$ \\
\hline Niagara Mohawk Power Corporation & $\begin{array}{l}\text { Nine Mile Point Nuclear Station, Unit } 1 \\
\text { Nine Mile Point Nuclear Station, Unit } 2\end{array}$ \\
\hline North Atlantic Energy Service Corporation & Seabrook Nuclear Power Station \\
\hline Northeast Utilities & $\begin{array}{l}\text { Haddam Neck (Connecticut Yankee) Plant } \\
\text { Millstone Nuclear Power Station, Units } 1 \text { \& } 2 \\
\text { Millstone Nuclear Power Station, Unit } 3\end{array}$ \\
\hline Northern States Power Company & $\begin{array}{l}\text { Monticello Nuclear Generating Plant } \\
\text { Pathfinder Atomic Plant } \\
\text { Prairie Island Nuclear Plant, Units } 1 \text { \& } 2\end{array}$ \\
\hline Omaha Public Power District & Fort Calhoun Station \\
\hline Pacific Gas \& Electric Company & $\begin{array}{l}\text { Diablo Canyon Nuclear Power Plant, Units } 1 \text { \& } 2 \\
\text { Humboldt Bay Power Plant, Unit } 3\end{array}$ \\
\hline PECO Energy Company & $\begin{array}{l}\text { Limerick Generating Station, Units } 1 \text { \& } 2 \\
\text { Peach Bottom Atomic Power Station, Unit } 1 \\
\text { Peach Bottom Atomic Power Station, Units } 2 \text { \& } 3\end{array}$ \\
\hline
\end{tabular}


Table 5 (continued)

\begin{tabular}{|c|c|}
\hline Operator & Plant name \\
\hline Pennsylvania Power \& Light Company & Susquehanna Steam Electric Station, Units 1 \& 2 \\
\hline Portland General Electric Company & Trojan Nuclear Plant \\
\hline Power Reactor Development Company & Fermi (Enrico) Atomic Power Plant, Unit 1 \\
\hline Public Service Company of Colorado & Fort St. Vrain Nuclear Generating Station \\
\hline Public Service Electric \& Gas Company & $\begin{array}{l}\text { Hope Creek Nuclear Generating Station } \\
\text { Salem Nuclear Generating Station, Units } 1 \& 2\end{array}$ \\
\hline Rochester Gas \& Electric Corporation & Ginna (Robert E.) Nuclear Power Plant \\
\hline Sacramento Municipal Utility District & Rancho Seco Nuclear Generating Station \\
\hline Saxton Nuclear Experimental Corporation & Saxton Nuclear Experimental Reactor Project \\
\hline South Carolina Electric \& Gas Company & Summer (Virgil C.) Nuclear Station \\
\hline Southern California Edison Company & $\begin{array}{l}\text { San Onofre Nuclear Generating Station, Unit } 1 \\
\text { San Onofre Nuclear Generating Station, Units } 2 \& 3\end{array}$ \\
\hline Southern Nuclear Operating Company (The) & $\begin{array}{l}\text { Farley (Joseph M.) Nuclear Plant, Units } 1 \& 2 \\
\text { Hatch (Edwin I.) Nuclear Plant, Units } 1 \& 2 \\
\text { Vogtle (Alvin W.) Nuclear Plant, Units } 1 \& 2\end{array}$ \\
\hline Tennessee Valley Authority & $\begin{array}{l}\text { Bellefonte Nuclear Plant, Units } 1 \& 2 \\
\text { Brown's Ferry Nuclear Power Station, Unit } 1 \\
\text { Brown's Ferry Nuclear Power Station, Units } 2 \text { \& } 3 \\
\text { Sequoyah Nuclear Plant, Units } 1 \& 2 \\
\text { Watts Bar Nuclear Plant, Unit } 1 \\
\text { Watts Bar Nuclear Plant, Unit } 2\end{array}$ \\
\hline Toledo Edison Company & Davis-Besse Nuclear Power Station \\
\hline TU Electric Company & Comanche Peak Steam Electric Station, Units $1 \& 2$ \\
\hline Union Electric Company & Callaway Plant \\
\hline Vermont Yankee Nuclear Power Corporation & Vermont Yankee Nuclear Power Station \\
\hline Virginia Electric and Power Company & $\begin{array}{l}\text { North Anna Power Station, Units } 1 \& 2 \\
\text { Surry Power Station, Units } 1 \& 2\end{array}$ \\
\hline
\end{tabular}


Table 5 (continued)

\begin{tabular}{ll}
\hline \multicolumn{1}{c}{ Operator } & \multicolumn{1}{c}{ Plant name } \\
\hline Washington Public Power Supply System & $\begin{array}{l}\text { WPPSS Nuclear Project, Unit 1 } \\
\text { WPPSS Nuclear Project, Unit } 2 \\
\text { WPPSS Nuclear Project, Unit 3 }\end{array}$ \\
Wisconsin Electric Power Company & Point Beach Nuclear Plant, Units 1 \& 2 \\
Wisconsin Public Service Corporation & Kewaunee Nuclear Power Plant \\
Wolf Creek Nuclear Operating Company & Wolf Creek Generating Station \\
Yankee Atomic Electric Company & Yankee Nuclear Power Station \\
\hline
\end{tabular}




\section{NUCLEAR POWER PLANT OPERATORS LISTED BY PLANT NAME}



Table 6. Nuclear power plant operators listed by plant

\begin{tabular}{|c|c|}
\hline Plant name & Operator \\
\hline $\begin{array}{l}\text { Arkansas Nuclear One, Units } 1 \& 2 \\
\text { Arnold (Duane) Energy Center }\end{array}$ & $\begin{array}{l}\text { Entergy Operations, Inc. } \\
\text { IES Utilities, Inc. }\end{array}$ \\
\hline Beaver Valley Power Station, Unit 1 & Duquesne Light Company \\
\hline Beaver Valley Power Station, Unit 2 & Duquesne Light Company \\
\hline Bellefonte Nuclear Plant, Units $1 \& 2$ & Tennessee Valley Authority \\
\hline Big Rock Point Nuclear Plant & Consumers Power Company \\
\hline Braidwood Station, Units $1 \& 2$ & Commonwealth Edison Company \\
\hline Brown's Ferry Nuclear Power Station, Unit 1 & Tennessee Valley Authority \\
\hline Brown's Ferry Nuclear Power Station, Units 2 \& 3 & Tennessee Valley Authority \\
\hline Brunswick Steam Electric Plant, Units 1 \& 2 & Carolina Power \& Light Company \\
\hline Byron Station, Units $1 \& 2$ & Commonwealth Edison Company \\
\hline Callaway Plant & Union Electric Company \\
\hline Calvert Cliffs Nuclear Power Plant, Units 1 \& 2 & Baltimore Gas \& Electric Company \\
\hline Catawba Nuclear Station, Unit 1 & Duke Power Company \\
\hline Catawba Nuclear Station, Unit 2 & Duke Power Company \\
\hline Clinton Power Station & Illinois Power Company \\
\hline Comanche Peak Steam Electric Station, Units 1 \& 2 & TU Electric Company \\
\hline Cook (Donald C.) Nuclear Power Plant, Units 1 \& 2 & Indiana Michigan Power Company \\
\hline Cooper Nuclear Station & Nebraska Public Power District \\
\hline Crystal River Nuclear Plant, Unit 3 & Florida Power Corporation \\
\hline Davis-Besse Nuclear Power Station & Toledo Edison Company \\
\hline Diablo Canyon Nuclear Power Plant, Units 1 \& 2 & Pacific Gas \& Electric Company \\
\hline Dresden Nuclear Power Station, Unit 1 & Commonwealth Edison Company \\
\hline Dresden Nuclear Power Station, Units $2 \& 3$ & Commonwealth Edison Company \\
\hline Farley (Joseph M.) Nuclear Plant, Units $1 \& 2$ & Southern Nuclear Operating Company (The) \\
\hline Fermi (Enrico) Atomic Power Plant, Unit 1 & Power Reactor Development Company \\
\hline Fermi (Enrico) Atomic Power Plant, Unit 2 & Detroit Edison Company \\
\hline Fitzpatrick (James A.) Nuclear Power Plant & New York Power Authority \\
\hline Fort Calhoun Station & Omaha Public Power District \\
\hline Fort St. Vrain Nuclear Generating Station & Public Service Company of Colorado \\
\hline Ginna (Robert E.) Nuclear Power Plant & Rochester Gas \& Electric Corporation \\
\hline Grand Gulf Nuclear Station & Entergy Operations, Inc. \\
\hline Haddam Neck (Connecticut Yankee) Plant & Northeast Utilities \\
\hline Harris (Shearon) Nuclear Power Plant & Carolina Power \& Light Company \\
\hline Hatch (Edwin I.) Nuclear Plant, Units 1 \& 2 & Southern Nuclear Operating Company (The) \\
\hline Hope Creek Nuclear Generating Station & Public Service Electric \& Gas Company \\
\hline Humboldt Bay Power Plant, Unit 3 & Pacific Gas \& Electric Company \\
\hline Indian Point, Unit 1 & Consolidated Edison Company of New York \\
\hline Indian Point, Unit 2 & Consolidated Edison Company of New York \\
\hline
\end{tabular}


Table 6 (continued)

Plant name

Indian Point, Unit 3

Kewaunee Nuclear Power Plant

LaCrosse (Genoa) Nuclear Generating Station

LaSalle County Station, Units 1 \& 2

Limerick Generating Station, Units 1 \& 2

Maine Yankee Atomic Power Station

McGuire (William B.) Nuclear Station, Units 1 \& 2

Millstone Nuclear Power Station, Units 1 \& 2

Millstone Nuclear Power Station, Unit 3

Monticello Nuclear Generating Plant

Nine Mile Point Nuclear Station, Unit 1

Nine Mile Point Nuclear Station, Unit 2

North Anna Power Station, Units 1 \& 2

Oconee Nuclear Station, Units 1-3

Oyster Creek Nuclear Power Plant

Palisades Nuclear Plant

Palo Verde Nuclear Generating Station, Units 1-3

Pathfinder Atomic Plant

Peach Bottom Atomic Power Station, Unit 1

Peach Bottom Atomic Power Station, Units 2 \& 3

Perry Nuclear Power Plant

Pilgrim Station

Point Beach Nuclear Plant, Units 1 \& 2

Prairie Island Nuclear Plant, Units $1 \& 2$

Quad Cities Station, Unịts 1 \& 2

Rancho Seco Nuclear Generating Station

River Bend Station

Robinson (H. B.) Plant, Unit 2

Salem Nuclear Generating Station, Units 1 \& 2

San Onofre Nuclear Generating Station, Unit 1

San Onofre Nuclear Generating Station, Units 2 \& 3

Saxton Nuclear Experimental Reactor Project

Seabrook Nuclear Power Station

Sequoyah Nuclear Plant, Units 1 \& 2

Shoreham Nuclear Power Station

South Texas Project, Units 1 \& 2

St. Lucie Plant, Unit 1
Operator

New York Power Authority

Wisconsin Public Service Corporation

Dairyland Power Cooperative

Commonwealth Edison Company

PECO Energy Company

Maine Yankee Atomic Power Company

Duke Power Company

Northeast Utilities

Northeast Utilities

Northern States Power Company

Niagara Mohawk Power Corporation

Niagara Mohawk Power Corporation

Virginia Electric and Power Company

Duke Power Company

GPU Nuclear Corporation

Consumers Power Company

Arizona Public Service Company

Northern States Power Company

PECO Energy Company

PECO Energy Company

Cleveland Electric Illuminating Company

Boston Edison Company

Wisconsin Electric Power Company

Northern States Power Company

Commonwealth Edison Company

Sacramento Municipal Utility District

Entergy Operations, Inc.

Carolina Power \& Light Company

Public Service Electric \& Gas Company

Southern California Edison Company

Southern California Edison Company

Saxton Nuclear Experimental Corporation

North Atlantic Energy Service Corporation

Tennessee Valley Authority

Long Island Lighting Company

Houston Lighting \& Power

Florida Power \& Light Company 
St. Lucie Plant, Unit 2

Summer (Virgil C.) Nuclear Station

Surry Power Station, Units 1 \& 2

Susquehanna Steam Electric Station, Units 1 \& 2

Three Mile Island Nuclear Station, Unit 1

Three Mile Island Nuclear Station, Unit 2

Trojan Nuclear Plant

Turkey Point Station, Unit 3 \& 4

Vallecitos Boiling Water Reactor

Vermont Yankee Nuclear Power Station

Vogtle (Alvin W.) Nuclear Plant, Units 1 \& 2

Waterford Generating Station, Unit 3

Watts Bar Nuclear Plant, Unit 1

Watts Bar Nuclear Plant, Unit 2

Wolf Creek Generating Station

WPPSS Nuclear Project, Unit 1

WPPSS Nuclear Project, Unit 2

WPPSS Nuclear Project, Unit 3

Yankee Nuclear Power Station

Zion Nuclear Plant, Units 1 \& 2
Florida Power \& Light Company

South Carolina Electric \& Gas Company

Virginia Electric and Power Company

Pennsylvania Power \& Light Company

GPU Nuclear Corporation

GPU Nuclear Corporation

Portland General Electric Company

Florida Power \& Light Company

General Electric Company

Vermont Yankee Nuclear Power Corporation

Southern Nuclear Operating Company (The)

Entergy Operations, Inc.

Tennessee Valley Authority

Tennessee Valley Authority

Wolf Creek Nuclear Operating Company

Washington Public Power Supply System

Washington Public Power Supply System

Washington Public Power Supply System

Yankee Atomic Electric Company

Commonwealth Edison Company 


\section{NUCLEAR POWER PLANTS LISTED BY STATE}




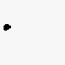


Table 7. Nuclear power plants by state

Alabama

Arizona

Arkansas

Califomia

Colorado

Connecticut

Florida

Georgia

Illinois

lowa

Kansas

Louisiana
Bellefonte Nuclear Plant, Units 1 \& 2

Brown's Ferry Nuclear Power Station, Unit 1

Brown's Ferry Nuclear Power Station, Units 2 \& 3

Farley (Joseph M.) Nuclear Plant, Units 1 \& 2

Palo Verde Nuclear Generating Station, Units 1-3

Arkansas Nuclear One, Units 1 \& 2

Diablo Canyon Nuclear Power Plant, Units 1 \& 2

Humboldt Bay Power Plant, Unit 3

Rancho Seco Nuclear Generating Station

San Onofre Nuclear Generating Station, Unit 1

San Onofre Nuclear Generating Station, Units 2 \& 3

Vallecitos Boiling Water Reactor

Fort St. Vrain Nuclear Generating Station

Haddam Neck (Connecticut Yankee) Plant

Millstone Nuclear Power Station, Units 1 \& 2

Millstone Nuclear Power Station, Unit 3

Crystal River Nuclear Plant, Unit 3

St. Lucie Plant, Unit 1

St. Lucie Plant, Unit 2

Turkey Point Station, Unit 3 \& 4

Hatch (Edwin I.) Nuclear Plant, Units 1 \& 2

Vogtle (Alvin W.) Nuclear Plant, Units 1 \& 2

Braidwood Station, Units 1 \& 2

Byron Station, Units 1 \& 2

Clinton Power Station

Dresden Nuclear Power Station, Unit 1

Dresden Nuclear Power Station, Units 2 \& 3

LaSalle County Station, Units 1 \& 2

Quad Cities Station, Units 1 \& 2

Zion Nuclear Plant, Units 1 \& 2

Arnold (Duane) Energy Center

Wolf Creek Generating Station

River Bend Station

Waterford Generating Station, Unit 3 
Table 7 (continued)

\begin{tabular}{|c|c|}
\hline State & Plant name \\
\hline Maine & Maine.Yankee Atomic Power Station \\
\hline Maryland & Calvert Cliffs Nuclear Power Plant, Units 1 \& 2 \\
\hline \multirow[t]{2}{*}{ Massachusetts } & Pilgrim Station \\
\hline & Yankee Nuclear Power Station \\
\hline \multirow[t]{5}{*}{ Michigan } & Big Rock Point Nuclear Plant \\
\hline & Cook (Donald C.) Nuclear Power Plant, Units 1 \& 2 \\
\hline & Fermi (Enrico) Atomic Power Plant, Unit 1 \\
\hline & Fermi (Enrico) Atomic Power Plant, Unit 2 \\
\hline & Palisades Nuclear Plant \\
\hline \multirow[t]{2}{*}{ Minnesota } & Monticello Nuclear Generating Plant \\
\hline & Prairie Island Nuclear Plant, Units 1 \& 2 \\
\hline Mississippi & Grand Gulf Nuclear Station \\
\hline Missouri & Callaway Plant \\
\hline \multirow[t]{2}{*}{ Nebraska } & Cooper Nuclear Station \\
\hline & Fort Calhoun Station \\
\hline New Hampshire & Seabrook Nuclear Power Station \\
\hline \multirow[t]{3}{*}{ New Jersey } & Hope Creek Nuclear Generating Station \\
\hline & Oyster Creek Nuclear Power Plant \\
\hline & Salem Nuclear Generating Station, Units 1 \& 2 \\
\hline \multirow[t]{8}{*}{ New York } & Fitzpatrick (James A.) Nuclear Power Plant \\
\hline & Ginna (Robert E.) Nuclear Power Plant \\
\hline & Indian Point, Unit 1 \\
\hline & Indian Point, Unit 2 \\
\hline & Indian Point, Unit 3 \\
\hline & Nine Mile Point Nuclear Station, Unit 1 \\
\hline & Nine Mile Point Nuclear Station, Unit 2 \\
\hline & Shoreham Nuclear Power Station \\
\hline \multirow[t]{3}{*}{ North Carolina } & Brunswick Steam Electric Plant, Units $1 \& 2$ \\
\hline & Harris (Shearon) Nuclear Power Plant \\
\hline & McGuire (William B.) Nuclear Station, Units 1 \& 2 \\
\hline \multirow[t]{2}{*}{ Ohio } & Davis-Besse Nuclear Power Station \\
\hline & Perry Nuclear Power Plant \\
\hline
\end{tabular}




\begin{tabular}{|c|c|}
\hline State & Plant name \\
\hline Oregon & Trojan Nuclear Plant \\
\hline \multirow[t]{9}{*}{ Pennsylvania } & Beaver Valley Power Station, Unit 1 \\
\hline & Beaver Valley Power Station, Unit 2 \\
\hline & Limerick Generating Station, Units 1 \& 2 \\
\hline & Peach Bottom Atomic Power Station, Unit 1 \\
\hline & Peach Bottom Atomic Power Station, Units 2 \& 3 \\
\hline & Saxton Nuclear Experimental Reactor Project \\
\hline & Susquehanna Steam Electric Station, Units $1 \& 2$ \\
\hline & Three Mile Island Nuclear Station, Unit 1 \\
\hline & Three Mile Island Nuclear Station, Unit 2 \\
\hline \multirow[t]{5}{*}{ South Carolina } & Catawba Nuclear Station, Unit 1 \\
\hline & Catawba Nuclear Station, Unit 2 \\
\hline & Oconee Nuclear Station, Units 1-3 \\
\hline & Robinson (H. B.) Plant, Unit 2 \\
\hline & Summer (Virgil C.) Nuclear Station \\
\hline South Dakota & Pathfinder Atomic Plant \\
\hline \multirow[t]{3}{*}{ Tennessee } & Sequoyah Nuclear Plant, Units $1 \& 2$ \\
\hline & Watts Bar Nuclear Plant, Unit 1 \\
\hline & Watts Bar Nuclear Plant, Unit 2 \\
\hline \multirow[t]{2}{*}{ Texas } & Comanche Peak Steam Electric Station, Units $1 \& 2$ \\
\hline & South Texas Project, Units $1 \& 2$ \\
\hline Vermont & Vermont Yankee Nuclear Power Station \\
\hline \multirow[t]{2}{*}{ Virginia } & North Anna Power Station, Units $1 \& 2$ \\
\hline & Surry Power Station, Units $1 \& 2$ \\
\hline \multirow[t]{3}{*}{ Washington } & WPPSS Nuclear Project, Unit 1 \\
\hline & WPPSS Nuclear Project, Unit 2 \\
\hline & WPPSS Nuclear Project, Unit 3 \\
\hline \multirow[t]{3}{*}{ Wisconsin } & Kewaunee Nuclear Power Plant \\
\hline & LaCrosse (Genoa) Nuclear Generating Station \\
\hline & Point Beach Nuclear Plant, Units 1 \& 2 \\
\hline
\end{tabular}





\section{OWNERSHIP NOTES}


Table 8. Ownership notes

\section{Utility}

Baltimore Gas \& Electric Company

Delmarva Power \& Light Company

Edison International

Entergy Arkansas, Inc.

Entergy Gulf States, Inc.

Entergy Louisiana, Inc.

మ)

GPU, Inc.

Houston Industries, Inc.

Houston Lighting \& Power

IES Industries, Inc.

Illinova Corporation

lowa-Illinois Gas \& Electric Company

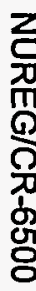

Jersey Central Power \& Light Company

Kansas City Power \& Light Company

Metropolitan Edison Company
Comments

Merger with Potomac Electric Power Company to form Constellation Energy Corp. in process.

Merger with Allantic Energy, Inc. in process.

Formerly SCEcorp.

Formerly Arkansas Power \& Light Company.

Formerly Gulf States Utilities Company.

Formerly Louisiana Power \& Light Company.

Formerly General Public Utilities Corporation.

Operating company being formed to operate South Texas Project.

Operating company being formed to operate South Texas Project.

Merger with Wisconsin Power \& Light and Interstate Power Company to become Interstate Energy Corp. in process.

Merger with Soyland Power Cooperative, Inc., in process.

Merged with Midwest Resources, Inc., to become MidAmerican Energy Company.

Part of consortium with Metropolitan Edison and Pennsylvania Electric known as GPU Energy.

Merger with UtiliCorp. in process. Western Resources, Inc., also making purchase bid with stockholders.

Part of consortium with Jersey Central Power \& Light and Pennsylvania Electric known as GPU Energy. 
MidAmerican Energy Company

Northern States Power Company

Pennsylvania Electric Company

Piedmont Municipal Power Agency

Portland General Electric Company

Puget Sound Power \& Light Company

$\$$ Soyland Power Cooperative, Inc.

Virginia Electric and Power Company

Washington Water Power Company

Western Resources, Inc.

Wisconsin Energy Corporation

Wisconsin Power \& Light Company

WPL Holdings, Inc.
Formed by merger of lowa-lllinois Gas \& Electric Company and Midwest Resources, Inc. MidAmerican Energy Holdings Company being formed.

Merger with Wisconsin Energy Corporation to become Primergy Corporation in process.

Part of consortium with Jersey Central Power \& Light and Metropolitan Edison known as GPU Energy.

Piedmont is offering to sell their $25 \%$ share of Catawba, Unit 2.

Merger with Enron in process.

Merger with Washington Energy Company in process.

Merger with Illinova Corporation in process.

Conducts business under the name of Virginia Power.

Merger with Sierra Pacific Resources to become Altus Corporation in process.

Trying to acquire Kansas City P\&L which is in merger process with UtiliCorp.

Merger with Northern States Power to become Primergy Corporation in process.

Merger with IES Industries, Inc. and Interstate Power Company to become Interstate Energy Corp. in process. 


\section{REFERENCES}

1. 1994 Production Costs - Operating Steam Electric Plants, UDI-2011-95, Utility Data Institute, September 1995.

2. Nuclear Reactors Built, Being Built, or Planned: 1994, DOE/OSTI-8200-R58, U.S. Department of Energy, Office of Nuclear Energy, Science and Technology, August 1995.

3. Electrical World Directory of Electric Power Producers, 104th Edition, McGraw-Hill, 1996.

4. "World List of Nuclear Power Plants", Nuclear News, p. 29, March 1996. 
NUREG/CR-6500

ORNL/TM-13297

Dist. Categories AN, CS, XG, 1S, 9L

\section{INTERNAL DISTRIBUTION}

$\begin{aligned} \text { 1. } & \text { J. J. Blass } \\ 2 . & \text { W. G. Craddick } \\ \text { 3. } & \text { C. W. Forsberg } \\ \text { 4. } & \text { E. C. Fox } \\ \text { 5. } & \text { R. G. Gilliland } \\ \text { 6. } & \text { S. R. Greene } \\ \text { 7. } & \text { S. A. Hodge } \\ 8-17 . & \text { C. R. Hudson } \\ \text { 18. } & \text { G. T. Mays } \\ \text { 19. } & \text { G. E. Michaels } \\ 20 . & \text { J. W. Miller } \\ 21 . & \text { D. L. Moses } \\ 22 . & \text { A. B. Poole } \\ 23 . & \text { W. P. Poore }\end{aligned}$
24. C. E. Pugh
25. R. L. Reid
26. J. J. Robinson
27. R. E. Schilling
28. J. O. Stiegler
29-38. V. S. White
39. K. A. Williams
40. Central Research Library
41. ORNL Y-12 Research Library Document Reference Section
42-43. Laboratory Records Department
44. Laboratory Records, ORNL
45. ORNL Patent Office

\section{EXTERNAL DISTRIBUTION}

46. C. Bergesen, Utility Data Institute, Inc., $1700 \mathrm{~K}$ Street, Suite 400 , Washington, D.C. 20006

47. G. Booras, Electric Power Research Institute, P.O. Box 10412, Palo Alto, CA 94303-0813

48. R. C. Brady, Office of Nuclear Reactor Regulation, U.S. Nuclear Regulation Commission, MS 011 H21, Washington, D.C. 20555-0001.

49. R. T. Eynon, Energy Information Administration, U.S. Department of Energy, 1000 Independence Avenue, Washington, D.C. 20585

50. M. Fertel, Nuclear Energy Institute, 1776 I Street MW, Suite 400, Washington, D. C. $20006-3708$

51. S. M. Franks, Office of Nuclear Energy, Science and Technology, U.S. Department of Energy, Washington, D.C. 20585

52. J. C. Geidl, Energy Information Administration, U.S. Department of Energy, 1000 Independence Avenue, Washington, D.C. 20585

53. M. J. Hutzler, Energy Information Administration, U.S. Department of Energy, 1000 Independence Avenue, Washington, D.C. 20585

54. R. L. Jones, VP Nuclear Power, Electric Power Research Institute, P.O. Box 10412, Palo Alto, CA 94303-0813

55. M. Knapik, McGraw-Hill Company, 1200 G Street NW, Suite 1100, Washington, D.C. 20005

56. T. R. Lash, Office of Nuclear Energy, Science and Technology, U.S. Department of Energy, Washington, D.C. 20585 
57. J. P. Mulkey, Office of Nuclear Energy, Science and Technology, U.S. Department of Energy, Washington, D.C. 20585

58. B. K. O'Brien, Energy Information Administration, U.S. Department of Energy, 1000 Independence Avenue, Washington D.C. 20585

59. M. Ryan, McGraw-Hill Company, $1200 \mathrm{G}$ Street NW, Suite 1100, Washington, D.C. 20005

60. G. E. Sliter, Electric Power Research Institute, P.O. Box 10412, Palo Alto, CA 94303-0813

61. J. M. Stamos, 317 Hart Senate Office Bldg., Washington, D.C. 20510-3305

62. R. W. Wood, Office of Nuclear Reactor Regulation, U.S. Nuclear Regulatory Commission MS 011 D23, Washington, D.C. 20555-0001

63. Office of the ORNL Site Manager, Department of Energy, Oak Ridge National Laboratory, P.O. Box 2008, Oak Ridge, TN 37831

64-65. Office of Scientific and Technical Information, P.O. Box 62, Oak Ridge, TN 37831 
2. TITLE AND SUBTITLE

Owners of Nuclear Power Plants

NUREG/CR-6500

ORNLTM-13297

3. DATE REPORT PUBLISHED

\begin{tabular}{l|l} 
MONTH & YEAR
\end{tabular}

November 1996

4. FIN OR GRANT NUMBER

J2411

5. AUTHOR(S)

C. R. Hudson, V. S. White

Technical

7. PERIOD COVERED (Inclusive Dates)

8. PERFORMING ORGANIZATION - NAME AND ADDRESS (HNRC, provide Division, Office or Region, U.S. Nuctear Regulatory Commission, and mailing address; if contractor, provide nemo and malling eddrass.)

Oak Ridge National Laboratory

Managed by Lockheed Martin Energy Research Corporation

P.O. Box 2009

Oak Ridge, TN 37831-8038

9. SPONSORING ORGANIZATION - NAME AND ADDRESS If NRC, type "Same as above"; if contracto, provide NRC Division, Office or Region, U.S. Nuclear Regulatory Commission, and mailing address.)

\section{Division of Reactor Program Management}

Office of Nuclear Reactor Regulation

U.S. Nuclear Regulatory Commission

Washington, DC 20555-0001

10. SUPPLEMENTARY NOTES

R. Wood, NRC Project Manager

11. ABSTRACT (200 words orless)

Commercial nuclear power plants in this country can be owned by a number of separate entities, each with varying ownership portions. Each of these owners may, in tum, have a parent/subsidiary relationship to other companies. In addition, the operator of the plant may be a different entity as well. This report provides a compilation on the owners/operators for all commercial power reactors in the United States. While the utility industry is currently experiencing changes in organizational structure which may affect nuclear plant ownership, the data in this report is current as of July 1996. The report is divided into the following sections representing different aspects of nuclear plant ownership:

- Nuclear power plant percentage ownership ordered by plant name

- Nuclear power plant percentage ownership ordered by utility name

- Utility/company relationships ordered by parent/holding company

- Utility/company relationships ordered by subsidiary

- Nuclear power plants listed by operator

- Nuclear power plant operators listed by plant name

- Nuclear power plants listed by state

12. KEY WORDS/DESCRIPTORS (List words or phrases that will assist researchers in locating the report)

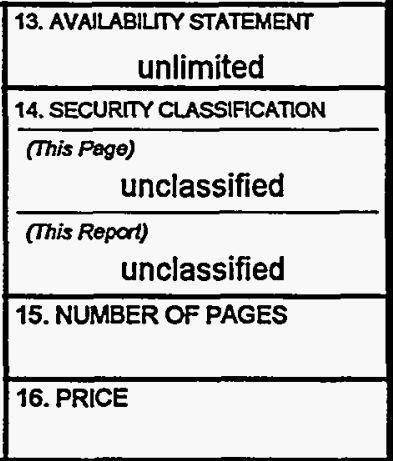

Nuclear Power Plants

Owners (ownership)

Utilities

Operators

Holding Companies 


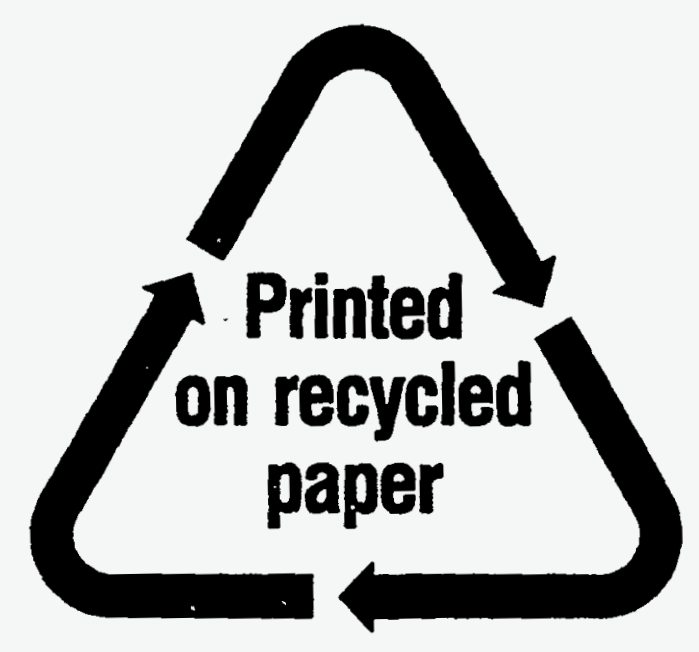

Federal Recycling Program 